This is the peer reviewed version of the following article: Rajić J, Dinić $S$, Uskoković $A$, Arambašić Jovanović J, Tolić $A$, Đorđević $M$, Đorđević $M$, Poznanović G, Mihailović M, Inic-Kanada A, Barisani-Asenbauer T, Grdović N, Vidaković M. DNA methylation of miR-200 clusters promotes epithelial to mesenchymal transition in human conjunctival epithelial cells. Exp Eye Res. 2020;197:108047. http://dx.doi.org/10.1016/j.exer.2020.108047

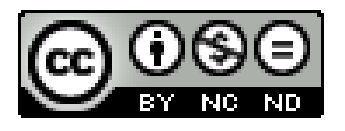

(C) 2020 Elsevier Ltd 


\section{DNA methylation of miR-200 clusters promotes epithelial to mesenchymal transition in human conjunctival epithelial cells}

Jovana Rajića, Svetlana Dinića , Aleksandra Uskokovića, Jelena Arambašić Jovanovića , Anja Tolića, Marija Đorđevića, Miloš Đorđevića , Goran Poznanovića , Mirjana Mihailovića, Aleksandra Inic-Kanadab, Talin Barisani-Asenbauer ${ }^{*}$, Nevena Grdovića ${ }^{*}$ and Melita Vidakovića

a Department of Molecular Biology, Institute for Biological Research "Siniša Stanković", National Institute of Republic of Serbia, University of Belgrade, Bulevar despota Stefana 142, 11060 Belgrade, Serbia

${ }^{b}$ Institute of Specific Prophylaxis and Tropical Medicine, Medical University of Vienna, Kinderspitalgasse 15, 1090 Vienna, Austria

c OCUVAC - Center of Ocular Inflammation and Infection, Laura Bassi Centres of Expertise, Medical University of Vienna, Kinderspitalgasse 15, 1090 Vienna, Austria

*Corresponding authors:

Dr. Nevena Grdović

Department of Molecular Biology, Institute for Biological Research "Siniša Stanković", National Institute of Republic of Serbia, University of Belgrade, Bulevar despota Stefana 142, 11060 Belgrade, Serbia, e-mail: nevenag@ibiss.bg.ac.rs

Dr. Talin Barisani-Asenbauer

OCUVAC - Center of Ocular Inflammation and Infection, Laura Bassi Centres of Expertise, Medical University of Vienna, Kinderspitalgasse 15, 1090 Vienna, Austria, e-mail:

talin.barisani@meduniwien.ac.at 


\begin{abstract}
Epithelial to mesenchymal transition (EMT) contributes to fibrosis associated pathologies including scarring of different ocular tissues. Recently targeting EMT is seen as an appropriate therapeutic approach for different fibrosis related eye diseases such as macular degeneration or glaucoma surgery related fibrosis. Nevertheless, for ocular surface diseases, target genes specific for particular cell type or condition are still undefined. This study aimed to expose the complex regulatory mechanisms that trigger EMT in human conjunctival epithelial (HCjE) cells. EMT was induced by prolonged treatment with two TGF- $\beta$ isoforms, TGF- $\beta 1$ and TGF- $\beta 2$, and their combination. TGF- $\beta 1$ showed the strongest potential for initiating EMT in HCjE cells, reflected on morphological changes, cell migration and the levels of mRNA expression of different epithelial ( CDH1, OCLN, DSP) and mesenchymal (CDH2, FN1, VIM, SNAI1, ZEB2, TWIST1) marker genes. Co-treatment with the DNA demethylating agent 5-Azacytidine (5-AzaC) was capable of stopping the transition of HCjE cells towards a mesenchymal phenotype, based on morphological features, reduced cell mobility and mRNA and protein expression levels of epithelial and mesenchymal marker genes. An EMT qRT-PCRbased array revealed that EMT induced considerable alterations in gene expression, with downregulation of the majority of epithelial marker genes and upregulation of genes specific for the mesenchymal state. The major effect of 5-AzaC treatment was observed as a suppression of mesenchymal marker genes, suggesting the involvement of upstream negative regulator(s) whose promoter demethylation and subsequent expression will in turn promote EMT switch off. The expression level of miRNAs potentially important for EMT induction was determined using qRT-PCRbased array which pointed at members of miR-200 family as main regulators of EMT process in HCjE cells. 5-AzaC treatment induced increased expression of miR-200a, -200b, -200c and miR-141 towards the control level, indicating important role of DNA methylation in their regulation. The DNA methylation status of both miR-200 family clusters, analyzed with high-resolution melting (HRM) and bisulfite sequencing (Bis-Seq), revealed that TGF- $\beta 1$-induced EMT was accompanied by increase in promoter $\mathrm{CpG}$ methylation of both miR-200 loci, which was reverted after 5-AzaC treatment. In conclusion, our results indicate that DNA demethylation of promoters of miR-200 loci is critically important for stopping and reverting the EMT in human conjunctival epithelial cells, suggesting the potential for the development of novel epigenetic-based therapeutic strategies for treating conjunctival conditions associated with EMT.
\end{abstract}

\title{
Keywords
}

Epithelial to mesenchymal transition (EMT); conjunctival fibrosis; human conjunctival epithelial cells (HCjE); TGF- $\beta$; 5-Azacytidine (5-AzaC); miRNAs; miR-200 family; DNA methylation

\section{Abbreviations}

EMT - epithelial to mesenchymal transition; HCjE - human conjunctival epithelial cells; TGF- $\beta$ transforming growth factor- $\beta$; 5-AzaC - 5-Azacytidine; Immunocytochemistry (ICC) 


\section{Intoduction}

Conjunctival fibrosis is the common possible consequence of different ocular inflammatory and infectious diseases globally. Although only a small proportion of affected individuals progress to the scarring stage, the sight threatening character of this complication causes significant morbidity and economic burden as in scarring trachoma, fibrosis associated with glaucoma filtration surgery (GFS), pterygium, ocular cicatricial pemphigoid (OCP) or complicated vernal or atopic keratoconjunctivitis (VKC, AKC) (Dale and Saban, 2015; Kechagia et al., 2016; Kim et al., 2016; Saw et al., 2011; Schlunck et al., 2016). Pathological processes that underlie above mentioned disorders are heterogeneous, still they are all characterized by irregular resolution of the healing processes that results in fibrosis. Mesenchymal cells which are involved in scarring originate from local fibroblast population and mesenchymal stem cells, but also from transdifferentiated epithelial cells derived from the process of epithelial to mesenchymal transition (EMT) (Micallef et al., 2012).

EMT is reversible process required for proper cell differentiation and organogenesis during development (Thiery et al., 2009). In adults, EMT plays important role in wound healing and pathophysiological processes such as organ fibrosis and cancer progression where it enables the first step of the metastatic route (Saitoh, 2018). During EMT, epithelial cells lose apical-basal polarity and cell-cell adhesions, and at the same time acquire the mesenchymal phenotype with the ability to migrate and to express mesenchymal cell markers. An important role of EMT in fibrotic processes has been thoroughly documented in kidney, liver and lung fibrosis (Rout-Pitt et al., 2018; Tennakoon et al., 2015), as well as in ocular fibrotic conditions (Ishikawa et al., 2015; Saika et al., 2009). With regard to conjunctival fibrosis, EMT was suggested to be involved in the pathogenesis of pterygium (Kato et al., 2007), and partially responsible for conjunctival and lacrimal gland fibrosis found in patients with chronic graft-versus-host disease (cGVHD) (Ogawa et al., 2009). In addition, infection of conjunctival epithelial cells with the bacteria Chlamydia trachomatis was shown to induce EMTlike changes that could contribute to the fibrotic process in trachomatous scarring (Rajic et al., 2017). It seems that EMT has wider implications then initially believed and that it contributes to almost every fibrotic condition (Stone et al., 2016). Therefore, potential therapeutic approaches in the treatment of various fibrosis-related diseases should consider blocking or even reversing the EMT in parallel with suppression of fibroblast activation.

TGF- $\beta$ proteins are considered as main EMT inducers as they initiate SMAD- and MAP kinasesignaling and downstream activation of EMT-related transcription factors (ZEB, SNAIL and TWIST) (Miyazono, 2009). The expression of EMT-related transcription factors results in downregulation of epithelial and at the same time, upregulation of mesenchymal marker genes responsible for maintaining the specific cell type phenotype (Lamouille et al., 2014). Besides transcriptional regulation, epigenetic machinery was suggested to be the master regulator of expression of all EMT related genes (Serrano-Gomez et al., 2016; Tam and Weinberg, 2013). Numerous studies have shown microRNAs (miRNAs) to be potent posttranscriptional regulators of the EMT. miRNAs are small noncoding RNAs that repress gene expression by binding to mRNA transcripts, leading to their degradation or inhibition of translation. Their number and specificity of the mechanism of action lie behind efficacy of miRNAs in gene repression. Namely, miRNAs establish multiple-to-multiple relationship with their targets, as it has been documented that one miRNA may regulate many 
genes, while one gene may be targeted by many miRNAs (Hashimoto et al., 2013). Through direct targeting of transcription factors associated with EMT and their targets, different miRNAs are considered to be the guardians of the epithelial phenotype and EMT suppressors that modulate the course and outcome of the EMT process (Zaravinos, 2015). DNA methylation is another epigenetic mechanism, usually associated with transcriptional repression, that has been related to EMT regulation. Although current opinion is that general hypomethylation and specific promoter hypermethylation of epithelial marker genes are the hallmarks of stable EMT in cancer cells (Pistore et al., 2017), the importance of DNA methylation for EMT induction remains elusive due to conflicting results obtained in different experimental models, depending on the cell type and EMT inducer (Choi et al., 2017; Pistore et al., 2017).

Given the increasing awareness of the importance of EMT in conjunctival fibrotic conditions and the complexity of the EMT regulatory network, the aim of this study was to investigate the multilayered regulatory mechanisms that trigger the switch from the epithelial to the mesenchymal phenotype in human conjunctival epithelial ( $\mathrm{HCjE}$ ) cells. The EMT model in $\mathrm{HCjE}$ cells was established by prolonged treatment with TGF- $\beta$ proteins under conditions that resemble chronic conjunctival inflammation associated with the fibrotic state. Unveiling the regulatory mechanisms and identification of critical participant(s) involved in the induction and progression of EMT could lay the foundation for development of novel strategies in therapy of conjunctival EMT-related fibrotic conditions.

\section{Materials and Methods}

\subsection{Cell culture and cell treatments}

The study was performed on the hTert-immortalized epithelial cell line obtained from normal human conjunctiva (HCjE) (Gipson et al., 2003; Rheinwald et al., 2002), kindly provided by Prof. llene Gipson from Schepens Eye Research Institute (Harvard Medical School, Boston). HCjE cells are transformed but not derived from cancer cells, which allowed us to perform a reproducible study with cells that have not undergone malignant transformation. HCjE cells were maintained under exponential growth in keratinocyte-serum free medium (Gibco, Carlsbad, CA) supplemented with $25 \mu \mathrm{g} / \mathrm{ml}$ bovine pituitary extract, $0.2 \mathrm{ng} / \mathrm{ml}$ recombinant human epidermal growth factor, $0.4 \mathrm{mM} \mathrm{CaCl}$, and $100 \mathrm{U} / \mathrm{ml}$ penicillin/streptomycin (Gibco) in a humidified (95\%) atmosphere and $5 \% \mathrm{CO}_{2}$ at $37^{\circ} \mathrm{C}$. For treatment with different TGF- $\beta$ isoforms, recombinant human TGF- $\beta 1$ (Gibco) and TGF- $\beta 2$ (SigmaAldrich, St. Louis, MO, USA) were added separately to the culture medium at the final concentration of $10 \mathrm{ng} / \mathrm{ml}$ or simultaneously at a final concentration of $5 \mathrm{ng} / \mathrm{ml}$ each. Medium containing TGF- $\beta$ proteins was changed every second day during six passages (approximately 30 days). For epigenetic drug treatments, $\mathrm{HCjE}$ cells were co-treated with the demethylation agent 5-Azacytidine (5-AzaC) (Sigma-Aldrich) at a concentration of $0.05 \mu \mathrm{M}$, which corresponds to $\mathrm{EC}_{80}$ for $\mathrm{HCjE}$ cells. The effects of 5-AzaC treatment with recovery period were examined in the experimental setup in which TGF$\beta 1$-treated HCjE cells were co-treated with 5-AzaC during the period between the second and third passage (for approximately 5 days) and then cultured in TGF- $\beta 1$ only until the end of the experiment (labeled as 5-AzaC R). For determination of the immediate effects of 5-AzaC, without a recovery period, TGF- $\beta 1$-treated $\mathrm{HCjE}$ cells were exposed to $5-\mathrm{AzaC}$ between the fifth and the last, sixth passage (labeled as 5-AzaC). During the 5-AzaC treatments, medium containing 5-AzaC and TGF- $\beta 1$ (final concentration of $10 \mathrm{ng} / \mathrm{ml}$ ) was changed every day. The entire experiment lasted six passages 
(approximately 30 days) during which TGF- $\beta 1$ was continuously applied. The morphology of the HCjE cells was monitored using an inverted microscope Nikon Eclipse TS100 (Nikon, Tokyo, Japan) and photos were taken using an attached Nikon Coolpix E5000 (Nikon, Tokyo, Japan) digital camera, before the first, third and sixth passage. All further isolations and analyses were performed on $\mathrm{HCjE}$ cells after the sixth passage unless otherwise indicated.

\subsection{Wound healing assay}

Control and treated $\mathrm{HCjE}$ cells were grown to confluence and a scratch was made through the cell monolayer with a $200 \mu \mathrm{l}$ sterile pipette tip. Cellular debris was removed by extensive washing with phosphate-buffered saline (PBS). To follow cell migration over time, images of the same field were taken at zero time and after $24 \mathrm{~h}$ using an inverted microscope Nikon Eclipse TS100 (Nikon, Tokyo, Japan) equipped with Nikon Coolpix E5000 (Nikon, Tokyo, Japan) digital camera. To determine the size of the open area between the cells on either side of the scratch, photos of five different fields per scratch obtained in three independent experiments were analyzed by a freeware ImageJ software ver. 1.52 (http://rsb.info.nih.gov/ii). The open area was expressed in pixels and the difference between $0 \mathrm{~h}$ and $24 \mathrm{~h}$ was calculated.

\subsection{Isolation of genomic DNA, mRNA and miRNA}

For isolation of genomic DNA and mRNA from control and treated HCjE cells, an AllPrep DNA/RNA Mini Kit (Qiagen, Hilden, Germany) was used according to the manufacturer's instructions. For isolation of miRNA from control and treated HCjE cells, a miRNeasy Mini Kit (Qiagen) was used according to the manufacturer's instructions. Total RNA was quantified by a NanoPhotometer N60/N50 (Implen GmbH, Munich, Germany).

\subsection{Quantitative real-time PCR (qRT-PCR)}

For cDNA synthesis, $1 \mu \mathrm{g}$ of the total RNA from control and treated HCjE cells was subjected to DNase I digestion and was reverse-transcribed with the RevertAid First Strand cDNA Synthesis Kit (Fermentas, Burlington, Canada) using a mix of random hexamer and oligo(dT) primers. mRNA levels were quantified using 2x Maxima SYBR Green/ROX qPCR Master Mix (Fermentas) on a QuantStudio 3 Real-Time PCR system (Applied Biosystems, Carlsbad, CA, USA). The thermal cycles consisted of initial denaturation at $95^{\circ} \mathrm{C} / 10 \mathrm{~min}$ and 40 cycles of two-step PCR at $95^{\circ} \mathrm{C} / 15 \mathrm{~s}$ and $60^{\circ} \mathrm{C} / 60 \mathrm{~s}$. The mRNA expression levels of the genes of interest were normalized to the mRNA expression level of the reference gene, glyceraldehyde 3-phosphate dehydrogenase (GAPDH). Relative fold-changes of the mRNA expression of targeted genes in treated $\mathrm{HCjE}$ cells compared to the control were determined by the 2-ddCT method (Livak and Schmittgen, 2001). Mean values and error bars are presented on log2 scales, while equivalent fold-change scales are represented on right $y$-axes of the graphs. Primer-BLAST (https://www.ncbi.nlm.nih.gov/tools/primer-blast/) was used to design the primers for human sequences stored in the GenBank under the following accession numbers: $C D H 1$ (E-cadherin, cadherin 1) NM_004360; OCLN (occludin) NM_002538; DSP (desmoplakin) NM_004415; CDH2 (N-cadherin, cadherin 2) NM_001792; FN1 (fibronectin 1) NM_212482; VIM (vimentin) NM_003380; SNAI1 (snail family transcriptional repressor 1) NM_005985; ZEB2 (zinc finger E-box binding homeobox 2) NM_014795; TWIST1 (twist family bHLH transcription factor 1) NM_000474; 
GAPDH (glyceraldehyde-3- phosphate dehydrogenase) NM_002046. The primers were validated by agarose gel electrophoresis to amplify one specific product, and their names and sequences are listed in Appendix A1.

2.5. Quantitative real-time PCR (qRT-PCR) based array analysis

2.5.1. Human Epithelial to Mesenchymal Transition $\mathrm{RT}^{2}$ Profiler $^{\mathrm{TM}} \mathrm{PCR}$ Array

The Human Epithelial to Mesenchymal Transition RT $^{2}$ Profiler ${ }^{\mathrm{TM}}$ PCR Array (PAHS-090ZA, Qiagen, Valencia, CA, USA) was used to analyze a set of 84 genes known to be involved in EMT process in control and treated $\mathrm{HCjE}$ cells. Reverse transcription was carried out with the RevertAid First Strand cDNA Synthesis Kit (Fermentas) on $0.5 \mu \mathrm{g}$ DNAse I-treated RNA, using a mix of random hexamer and oligo(dT) primers. Single stranded cDNA was mixed with 2x Maxima SYBR Green/ROX qPCR Master Mix (Fermentas) and qRT-PCR was performed on a QuantStudio 3 Real-Time PCR system (Applied Biosystems) following the manufacturer's recommendations. Data analyses were performed online at https://www.qiagen.com/it/shop/genes-and-pathways/data-analysis-center-overview-page/ webpage. The relative quantification of the transcripts was performed according to the 2-ddCt method after normalization against ACTB, B2M, GAPDH, HPRT1 and RPLPO mRNA expression levels. Genes exhibiting fold-changes in mRNA expression that were greater than two or were less than 0.5 as compared to the control were considered significant. Values are presented on log2 scales while equivalent fold-change scales are represented on right $y$-axes of the graphs. Arrays were validated in additional qRT-PCR experiments.

\subsection{2. miRCURY LNA miRNA Cancer Focus PCR Panel}

To determine the differentially expressed miRNAs between the control and treated HCjE cells, miRCURY LNA miRNA Cancer Focus PCR Panel (YAHS-102YA, Qiagen, Valencia, CA, USA) was used. A total of 10 ng of RNA was reverse transcribed to CDNA using the miRCURY LNA RT Kit (Qiagen, Hilden, Germany). In this step miRNA were polyadenylated and reverse transcribed using universal oligo-dT primers. Amplification of mature miRNA was performed using 2x miRCURY SYBR Green/ROX Master Mix on a QuantStudio 3 Real-Time PCR system (Applied Biosystems) according to the manufacturer's recommendations. Data analyses were performed online at https://www.qiagen.com/it/shop/genes-and-pathways/data-analysis-center-overview-page/. The relative fold-change of target miRNA was calculated using the 2-ddCt method, with ten miRNAs from the entire panel, with the best gNorm-calculated stability measures used for normalization. miRNAs that had fold-changes in expression that were greater than two or less than 0.5 as compared to the control were considered significant. Values are presented on log2 scales on left y-axes while equivalent fold-change scales are presented on right $y$-axes of the graphs.

\subsection{Immunoblot analysis}

For protein isolation, control and TGF- $\beta 1$-treated HCjE cells were lysed with radioimmunoprecipitation assay buffer (Sigma-Aldrich) in the presence of a protease inhibitor mixture (SERVA Electrophoresis $\mathrm{GmbH}$, Heidelberg, Germany) and incubated for $30 \mathrm{~min}$ on ice. The cell lysates were centrifuged for $20 \mathrm{~min}$ at $16,000 \mathrm{xg}$ at $4{ }^{\circ} \mathrm{C}$ and the total protein concentration in the supernatant was estimated by the Lowry assay (Lowry et al., 1951) using bovine serum albumin 
(BSA) as standard. Loading of equal amounts of proteins onto a $12 \%$ sodium dodecyl sulfate (SDS) polyacrylamide gel was followed by electrotransfer to a polyvinylidene difluoride membranes, blocking with PBS/5\% non-fat dry milk (Santa Cruz Biotechnology, Santa Cruz, CA, USA) for 60 min, and overnight incubation at $4{ }^{\circ}$ with primary antibodies. After incubation at room temperature for 60 min with secondary antibodies conjugated to horseradish peroxidase, the blots were stained using chemiluminescence according to the manufacturer's instructions (Amersham Pharmacia Biotech, Amersham, UK). Membranes were stripped by incubation with $100 \mathrm{mM} \beta$-mercaptoethanol, $2 \% \mathrm{SDS}, 62.5 \mathrm{mM}$ Tris- $\mathrm{HCl}\left(\mathrm{pH} \mathrm{6.7)}\right.$ at $55^{\circ} \mathrm{C}$ for $35 \mathrm{~min}$ and reprobed with anti-GAPDH antibody. Relative protein levels were determined by densitometry using TotalLab ver. 1.10 electrophoresis software (Phoretix International Ltd, Newcastle upon Tyne, UK) with normalization to the amount of GAPDH present in each sample. All antibodies, dilutions and manufacturers are listed in Appendix A2.

\subsection{Immunocytochemistry (ICC)}

HCjE cells were seeded on sterile glass coverslips in 24-well tissue culture plates and after reaching $70 \%$ confluence, the cells were fixed with 3.5\% formaldehyde (Zorka Pharma, Šabac, Serbia) in PBS for 10 min at $37{ }^{\circ} \mathrm{C}$, permeabilized with PBS/0.2\% Triton X-100 for 10 min and blocked with PBS/3\% $\mathrm{BSA}$ for $60 \mathrm{~min}$. To acquire the fluorescent signal, the slides were subsequently incubated overnight at $4{ }^{\circ} \mathrm{C}$ with primary and fluorescently labeled secondary antibodies, all listed in Appendix A2. Antibodies were diluted in PBS/0.2\% Tween-20, while all washes were done in PBS/0.2\% Tween-20. For visualization of DNA, incubation of slides with 4,6-diamidino-2-phenylindole (DAPI) (Roche Diagnostics, Mannheim, Germany) $(0.1 \mu \mathrm{g} / \mu \mathrm{l})$ for 5 min was performed. The cover slips were mounted on glass slides with Mowiol (Calbiochem, San Diego, CA, USA) and images were taken with an Axiocam digital camera attached to the Axio Observer Z1 microscope (Carl Zeiss Microscopy $\mathrm{GmbH}$, Jena, Germany).

\subsection{Flow cytometry}

For cytofluorimetric analysis of E-cadherin and N-cadherin expression, control and TGF- $\beta 1$-treated $\mathrm{HCjE}$ cells were suspended from growing cultures with PBS/0.05\% trypsin/0.02\% EDTA (Gibco) and resuspended in fresh medium to achieve recovery of surface antigens disturbed by trypsinisation. After 60 min at $37^{\circ} \mathrm{C}$, under $5 \% \mathrm{CO}_{2}$ and $95 \%$ humidity, the cells were permeabilized by resuspending in PBS/0.3\% saponin (Sigma-Aldrich) for $15 \mathrm{~min}$. After this step, the cells were incubated for $60 \mathrm{~min}$ with primary antibody and subsequently for $30 \mathrm{~min}$ with fluorescently labeled secondary antibody, all listed in Appendix A2. Antibodies were diluted in PBS/0.1\% saponin/3\% BSA (blocking), while all washes were performed in PBS/0.1\% saponin. Following the staining procedure, the cells were resuspended in culture medium and analyzed for surface marker expression on a FACS Aria III flow cytometer (BD Biosciences, San Jose, CA, USA), using FACSDiva software.

\subsection{DNA methylation analysis}

\subsubsection{Bisulfite conversion of DNA}

Genomic DNA from control and treated HCjE cells was bisulfite-converted using the EZ-DNA methylation kit (Zymo Research, Orange, CA, USA), according to the manufacturer's instructions. 
CpG islands in the $5^{\prime}$ flanking and coding region of the miRNAs were predicted using Methyl Primer Express ver. 1.0 software (Applied Biosystems, Carlsbad, CA, USA) by Davalos et al. (Davalos et al., 2012). Also, primers used for DNA methylation analysis of the hsa-miR-200b/a/429 locus were taken from Davalos et al. (Davalos et al., 2012), while primers used for the hsa-miR-200c/141 locus were designed in MethPrimer (http://www.urogene.org/cgi-bin/methprimer/methprimer.cgi). Two different forward primers and single reverse primer were used for high resolution melting (HRM) and bisulfite sequencing (Bis-Seq) for hsa-miR-200b/a/429, while the same pair of primers was used for hsa-miR-200c/141 for both DNA methylation analysis methods. The primers were validated by agarose gel electrophoresis to amplify one specific product, and their names and sequences are listed in Appendix A3. For the identification of putative transcription factor binding sites of SP1 and c-MYB in the promoter of miR-200b/a/429 and hsa-miR-200c/141, the on-line based tool Match ${ }^{\mathrm{TM}}$ public ver. 1.0 (http://www.gene-regulation.com/pub/programs.html\#match) was used with default parameters. Positions of Z-box (CAGGTA) and ZEB-type E-boxes (CACCTG) in the analyzed regions were identified by Burk et al. (Burk et al., 2008) and Bracken et al. (Bracken et al., 2008), respectively.

\subsubsection{High resolution melting (HRM)}

Human methylated and non-methylated DNA standards (D5014) (Zymo Research) were bisulfiteconverted using an EZ-DNA methylation kit (Zymo Research) according to the manufacturer's instructions and mixed at 0:100, 25:75, 50:50, 75:25 and 100:0 ratios to generate a range of methylated:non-methylated DNA standards. PCR amplification and HRM analysis were performed sequentially on a QuantStudio 3 Real-Time PCR system. The reaction mixture consisted of $5 \mu \mathrm{l} 2 \mathrm{x}$ MeltDoctor HRM Master Mix (Applied Biosystems), $0.3 \mu \mathrm{M}$ of each primer, and $2 \mu$ l bisulfiteconverted template (theoretical concentration of $10 \mathrm{ng} / \mu \mathrm{l}$ ). The temperature profile of the amplification was as follows: initial denaturation at $95^{\circ} \mathrm{C} / 10 \mathrm{~min}, 45$ cycles of three-step PCR and a final elongation step at $72{ }^{\circ} \mathrm{C} / 7 \mathrm{~min}$. The three-step PCR program consisted of 5 cycles of denaturation at $95^{\circ} \mathrm{C} / 30 \mathrm{~s}$, annealing at $62{ }^{\circ} \mathrm{C} / 30 \mathrm{~s}$ and elongation at $72{ }^{\circ} \mathrm{C} / 60 \mathrm{~s} ; 5$ cycles of denaturation at $95{ }^{\circ} \mathrm{C} / 30 \mathrm{~s}$, annealing at $58{ }^{\circ} \mathrm{C} / 30 \mathrm{~s}$ and elongation at $72{ }^{\circ} \mathrm{C} / 60 \mathrm{~s}$; and $35 \mathrm{cycles}$ of denaturation at $95^{\circ} \mathrm{C} / 30 \mathrm{~s}$, annealing at $55^{\circ} \mathrm{C} / 30 \mathrm{~s}$ and elongation at $72{ }^{\circ} \mathrm{C} / 60 \mathrm{~s}$. The HRM program performed fluorescence acquisition at each temperature increment of $0.025^{\circ} \mathrm{C} / \mathrm{s}$ between $60-95{ }^{\circ} \mathrm{C}$ and HRM Software v3.1 (Applied Biosystems) was used for end-product analysis. The level of methylation for each sample was calculated from the linear regression equation obtained after plotting normalized peak heights of DNA standards against the percentage of DNA methylation.

\subsubsection{Bisulfite sequencing (Bis-Seq)}

Amplification of bisulfite-modified DNA was carried out on an Eppendorf Mastercycler Pro (Eppendorf GmbH, Vienna, Austria). The PCR reaction mixture consisted of $15 \mu \mathrm{l} 2$ x AmpliTaq Gold 360 Master Mix (Applied Biosystems), $0.5 \mu \mathrm{M}$ of each primer, and $3 \mu \mathrm{l}$ of bisulfite-converted template (theoretical concentration of $15 \mathrm{ng} / \mu \mathrm{l})$. PCR cycling conditions were as follows: initial denaturation at $95{ }^{\circ} \mathrm{C} / 10 \mathrm{~min}, 60$ cycles of two-step PCR, and final elongation at $72{ }^{\circ} \mathrm{C} / 7 \mathrm{~min}$. The two-step PCR program consisted of 5 cycles of denaturation at $95^{\circ} \mathrm{C} / 30 \mathrm{~s}$, annealing at $58^{\circ} \mathrm{C} / 30 \mathrm{~s}$ and elongation at $72{ }^{\circ} \mathrm{C} / 60 \mathrm{~s}$; and 55 cycles of denaturation at $95^{\circ} \mathrm{C} / 30 \mathrm{~s}$, annealing at $55^{\circ} \mathrm{C} / 30 \mathrm{~s}$ 
and elongation at $72{ }^{\circ} \mathrm{C} / 60 \mathrm{~s}$. Purification of PCR products and Sanger sequencing of bisulfiteconverted DNA were carried out by Macrogen Europe B.V. (Amsterdam, Netherlands). BiQ Analyzer software (Max Planck Institute for Informatics, Saarbrücken, Germany) was used for sequence alignment.

\subsection{Statistical analysis}

Statistical analysis was conducted using IBM SPSS Statistics for Windows, ver. 20.0 (Armonk, NY, USA). Student's t-test was used to compare means of normally distributed variables between two groups. For comparison of more groups One-Way ANOVA was applied followed by Tukey's HSD test. For analysis of qRT-PCR data, to estimate statistical significance between the mean of the control group and the means of other groups, a One Sample t-test was applied on log2 transformed data. Mean values and error bars are presented on log2 scales while equivalent fold-change scales are shown on right $y$-axes of the graphs. The null hypothesis was rejected at $\mathrm{P}<0.05$. " $*$ " indicates a statistically significant difference compared to the control ( $* \mathrm{P}<0.05 ; * * \mathrm{P}<0.01)$; "\#” indicates a statistically significant difference compared to the TGF- $\beta 1$-treated $H C$ C E cells ( $\#<0.05$; \#\# $P<0.01$ ); "+" indicates a statistically significant difference compared to 5-AzaC R-treated HCjE cells (+ $\mathrm{P}<0.05$; $++P<0.01)$. Experiments were performed in triplicate unless otherwise indicated and all results are presented as the means \pm SEMs (standard error of the mean).

\section{Results}

3.1.TGF- $\beta 1$ possesses the highest potential for EMT induction in $\mathrm{HCjE}$ cells

TGF- $\beta$ proteins play an important role in ocular physiology from embryonic development to adulthood and in various pathological conditions. Ocular epithelial cells produce all three TGF- $\beta$ members, especially during inflammation or tissue repair process (Saika et al., 2009), but contribution of a particular TGF- $\beta$ isoform to a certain pathological state remains elusive. In order to assess differences in capability and potential of TGF- $\beta 1$ and TGF- $\beta 2$ to induce EMT in human conjunctival epithelial (HCjE) cells, $\mathrm{HCjE}$ cells were treated with TGF- $\beta 1$ and TGF- $\beta 2$ separately and in combination (Fig. 1). The cells were exposed to TGF- $\beta$ continually for six passages (approximately 30 days) with the aim of creating conditions resembling the state of chronic inflammation that precedes fibrotic changes in the tissue.

TGF- $\beta 1$ induced the most prominent change in cell morphology (Fig. 1A). Loss of tight cell-cell contacts was observed after the first passage, and in time the cells gradually changed their epithelial polygonal shape to an elongated spindle-like shape typical for the mesenchymal phenotype (Appendix B1). The combination of TGF- $\beta 1$ and TGF- $\beta 2$ triggered similar morphological changes, while the TGF- $\beta 2$ induced only alterations in cell-cell adhesion with the sporadic presence of spindlelike cells (Fig. 1A and Appendix B1). Morphological changes were associated with an acquired ability to move that was assessed using the wound healing assay (Fig. 1B). While all treatments reduced the "open wound area", the diminishment was statistically significant after treatment with TGF- $\beta 1$ and with a combination of TGF- $\beta$ proteins.

To further evaluate the effects of the applied treatments on EMT induction, the expression level of several marker genes was estimated using qRT-PCR (Fig. 1C). E-cadherin (CDH1), occludin (OCLN) and 
desmoplakin (DSP) were selected as typical epithelial cell markers whose expression is lost in cells undergoing EMT, whereas N-cadherin (CDH2), vimentin (VIM) and fibronectin (FN1) were used as key mesenchymal markers that are acquired during EMT (Serrano-Gomez et al., 2016; Zeisberg and Neilson, 2009). Also, the levels of expression of mRNAs encoding for three EMT-related transcription factors - SNAI1 (SNAI1), ZEB2 (ZEB2) and TWIST1 (TWIST1) were evaluated. The results of mRNA expression analysis in control and treated $\mathrm{HCjE}$ cells after the first and sixth passage are presented on Fig. $1 \mathrm{C}$ where the control level in the graphs is set on zero on the log2 scale, or set on 1 on the linear scale. The expression levels of $C D H 1$ and $O C L N$ remained near the control level after the first passage, while at the end of the treatment period (sixth passage), a statistically significant reduction in expression was observed in all treatment groups. Similar trends were obtained for DSP expression, but with statistical significance only for the combination of TGF- $\beta$ proteins after the sixth passage. Unlike epithelial markers, the expression levels of mesenchymal marker genes increased already after the first passage and later followed

A

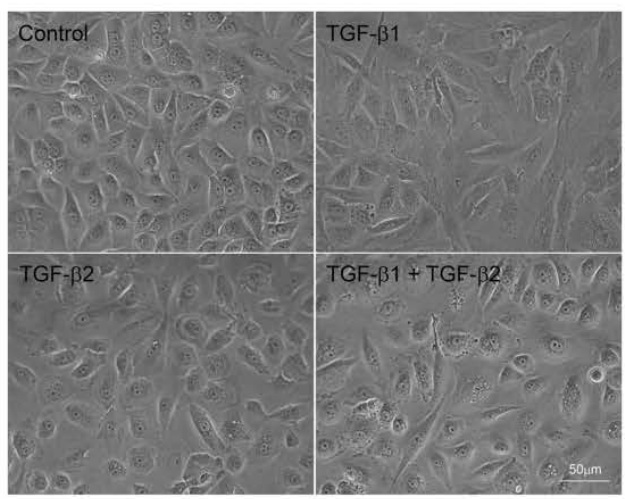

B

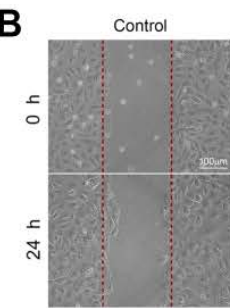

TGF- $\beta 1$

TGF- $\beta 2$

TGF- $\beta 1+$ TGF- $\beta 2$
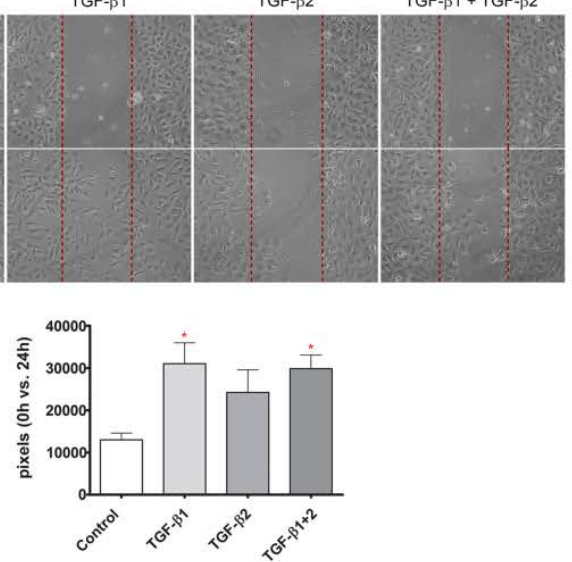

C
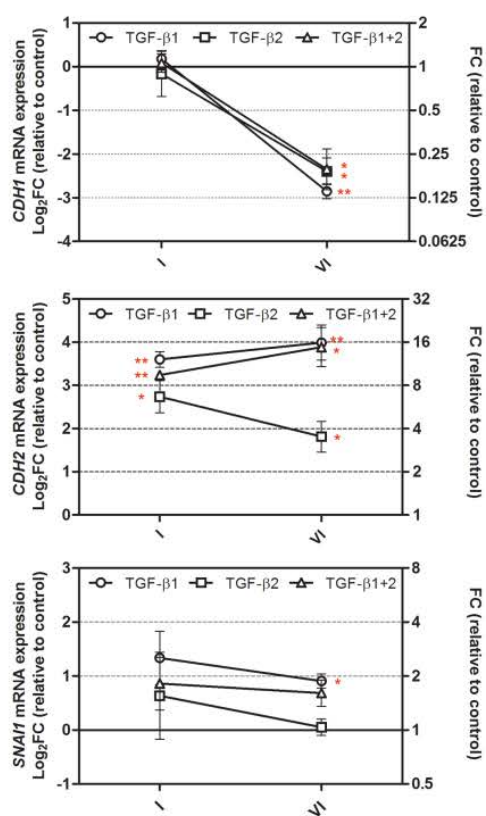
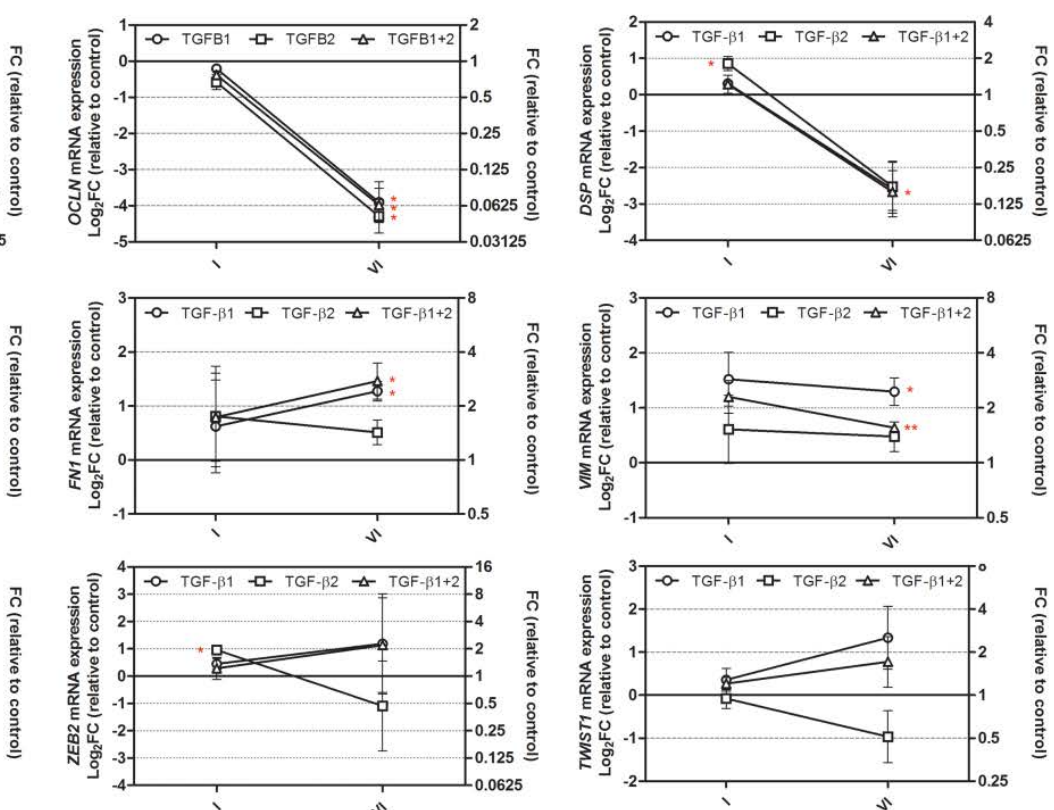

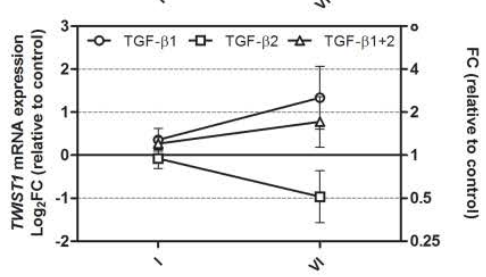

Fig 1. EMT induction in HCjE cells exposed to TGF- $\beta 1$, TGF- $\beta 2$ and a combination of TGF- $\beta$ proteins (TGF- $\beta 1+$ TGF- $\beta 2$ ) for six passages. (A) Morphological changes of HCjE cells after treatments with different TGF- $\beta$ isoforms. (B) Cell migration analysis of $\mathrm{HCjE}$ cells after treatments with different TGF- $\beta$ isoforms. Representative images of the in vitro wound healing 
assay at $0 \mathrm{~h}$ and $24 \mathrm{~h}$ are shown. The graph represent the area (in pixels) of the wound covered by cells after $24 \mathrm{~h}$. Data are shown as the means \pm SEMs from three independent experiments; ${ }^{*} P<0.05$ compared to the control. (C) mRNA levels of epithelial and mesenchymal marker genes after the first and the sixth passage in control and HCjE cells treated with different TGF- $\beta$ isoforms as determined by qRT-PCR. mRNA levels are relative to GAPDH and are expressed as the means of $\log 2$ fold change (treated vs. control) in three replicates \pm SEM. Positive log 2 fold changes indicate upregulation in treated cells as compared to the control, and negative log 2 fold changes indicate downregulation in treated cells as compared to the control. The equivalent fold change scale is presented on right $y$-axes. $* \mathrm{P}<0.05$ and ${ }^{* *} \mathrm{P}<0.01$ compared to the control.

A

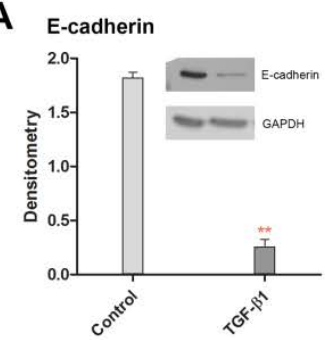

$$
\mathrm{N} \text {-cadherin }
$$

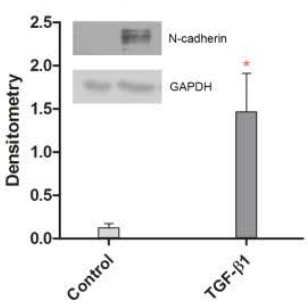

B
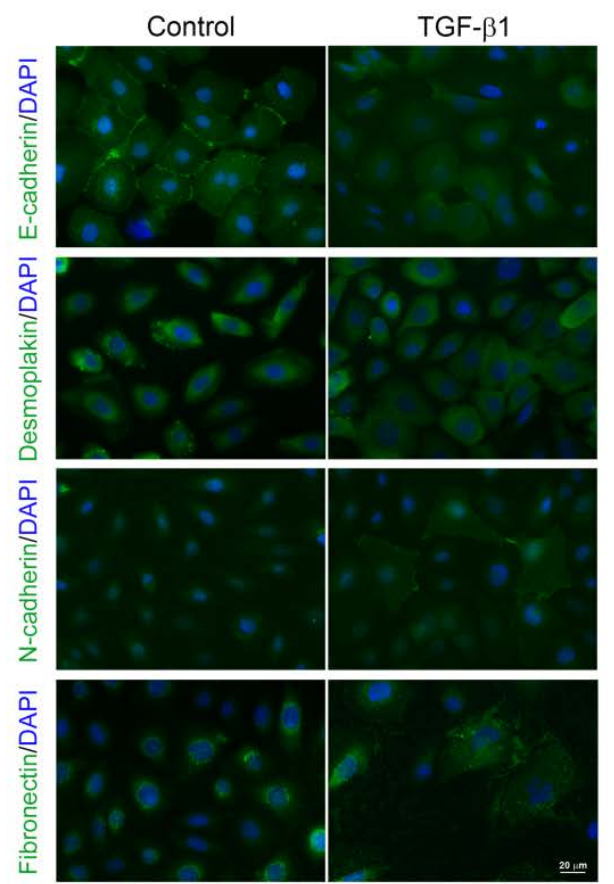

C

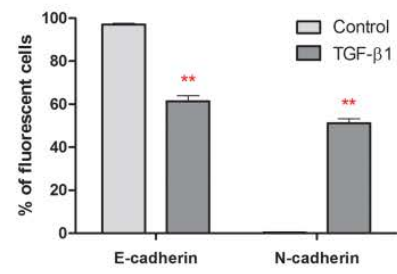

different kinetics depending on the applied treatment (Fig. 1C). Namely, TGF- $\beta 1$ induced the most pronounced changes in the expression levels of all mesenchymal marker genes and EMT-related transcription factors. The combination of TGF- $\beta$ proteins induced effects comparable to those observed after treatment with TGF- $\beta 1$ but to a lesser extent. Treatment with TGF- $\beta 2$ did not induce the expression of either ZEB2 or TWIST1, while the expression of the remaining analyzed genes increased to a level that was markedly lower than observed after the TGF- $\beta 1$ treatment (Fig. 1C). Those findings indicated that TGF- $\beta 1$ possesses the highest potential for EMT induction in $\mathrm{HCjE}$ cells.

Fig. 2. Changes in levels of expression of epithelial and mesenchymal marker proteins, in $\mathrm{HCjE}$ cells after six passages under the TGF- $\beta 1$ treatment. (A) Protein levels of epithelial (Ecadherin and desmoplakin) and mesenchymal (N-cadherin and fibronectin) marker proteins in control and TGF- $\beta 1$-treated HCjE cells determined by immunoblot analysis. The densities of the bands from three immunoblots are presented as the means \pm SEMs after normalization to GAPDH; * $\mathrm{P}<0.05$ and ** $P<0.01$ compared to the control. (B) Immunofluorescence analysis of control and TGF- $\beta 1$-treated HCjE cells with antibodies against epithelial (E-cadherin and desmoplakin) and mesenchymal ( $\mathrm{N}$-cadherin and fibronectin) marker proteins (green fluorescence). Nuclei are stained with DAPI (blue fluorescence). (C) Cytofluorometric assessment of the $\mathrm{E} / \mathrm{N}$ cadherin switch after TGF- $\beta 1$ treatment of HCjE cells.

Means \pm SEMs on the graph represents three measurements of the percentage of fluorescent cells; $* * \mathrm{P}<0.01$ compared to the control.

To confirm that the TGF- $\beta 1$-induced change in the gene expression profile is also replicated on the protein level, immunoblot analysis with two epithelial and two mesenchymal markers was performed (Fig. 2A). Treatment with TGF- $\beta 1$ led to a statistically significant and marked reduction in $\mathrm{E}$ -

cadherin and desmoplakin levels and to a statistically significant increase in the presence of $\mathrm{N}$ - 
cadherin and fibronectin as compared to the control. The same was confirmed by immunocytochemistry (Fig. 2B). The disappearance of discrete desmoplakin-positive clusters localized on the periphery of control cells and an intense fibronectin fiber network that surrounds the cells after treatment with TGF- $\beta 1$ was documented. Also, the complete loss of E-cadherin that was accompanied by the appearance of N-cadherin in TGF- $\beta 1$ treated cells was observed (Fig. 2B). $\mathrm{E} / \mathrm{N}$-cadherin switch, quantified using flow cytometry (Fig. 2C), confirmed that HCjE cells are susceptible to TGF- $\beta 1$ treatment which drove them towards the mesenchymal phenotype via the EMT process.

3.2. The demethylating agent 5-AzaC can stop EMT-related changes and partly restore the epithelial phenotype in $\mathrm{HCjE}$ cells

To examine the impact of DNA demethylation on TGF- $\beta 1$-induced EMT of human conjunctival cells, HCjE cells were treated with 5-Azacytidine (5-AzaC), a prototypal DNA methyltransferase (DNMT) inhibitor. Considering that DNMT inhibition results in loss of methylation marks during replication that becomes evident only after cell proliferation (Stresemann and Lyko, 2008), two different time points were selected for 5-AzaC application. In the first experimental setup 5-AzaC was applied in the middle of the experimental period allowing multiple cell divisions after drug application and detection of effects of DNA demethylation after the recovery period (labeled as 5-AzaC R). In the second experimental setup 5-AzaC was applied at the end of experimental period allowing identification of immediate effects of DNA demethylation, without a recovery period (marked as 5$A z a C)$. The effects of DNA demethylation were evaluated at the end of the experimental period, after the sixth passage, in both 5-AzaC treated samples. $\mathrm{HCjE}$ cells were highly sensitive to the 5AzaC treatment as $\mathrm{IC}_{50}$ was $0.175 \mu \mathrm{M}$ (results not shown), which could be related to their nonmalignant origin as the effects of DNMT1 inhibitors were mostly studied on cell lines derived from cancer cells. Bearing in mind that 5-AzaC exerts DNA demethylating effects via DNMT inhibition only at low concentrations, while high concentrations of 5-AzaC induce inhibition of cell proliferation and cytotoxicity (Agrawal et al., 2018), the dose of $0.05 \mu \mathrm{M}$ representing $\mathrm{IC}_{80}$ was applied to $\mathrm{HCjE}$ cells. 
A

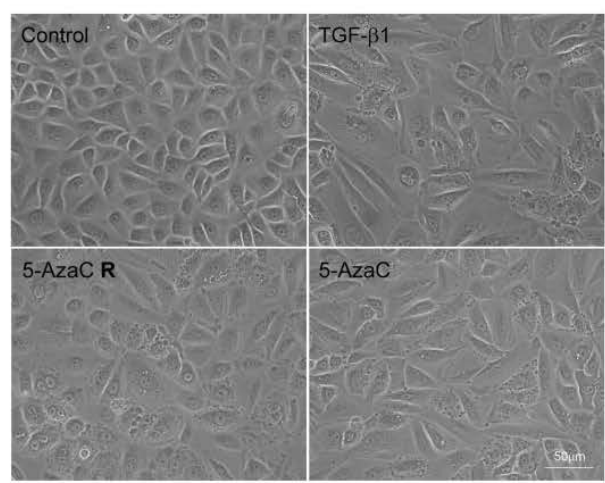

B
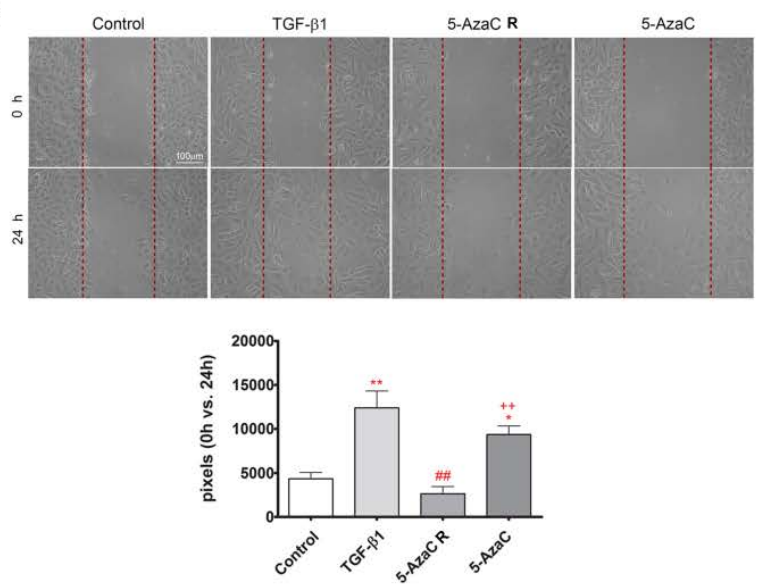

C

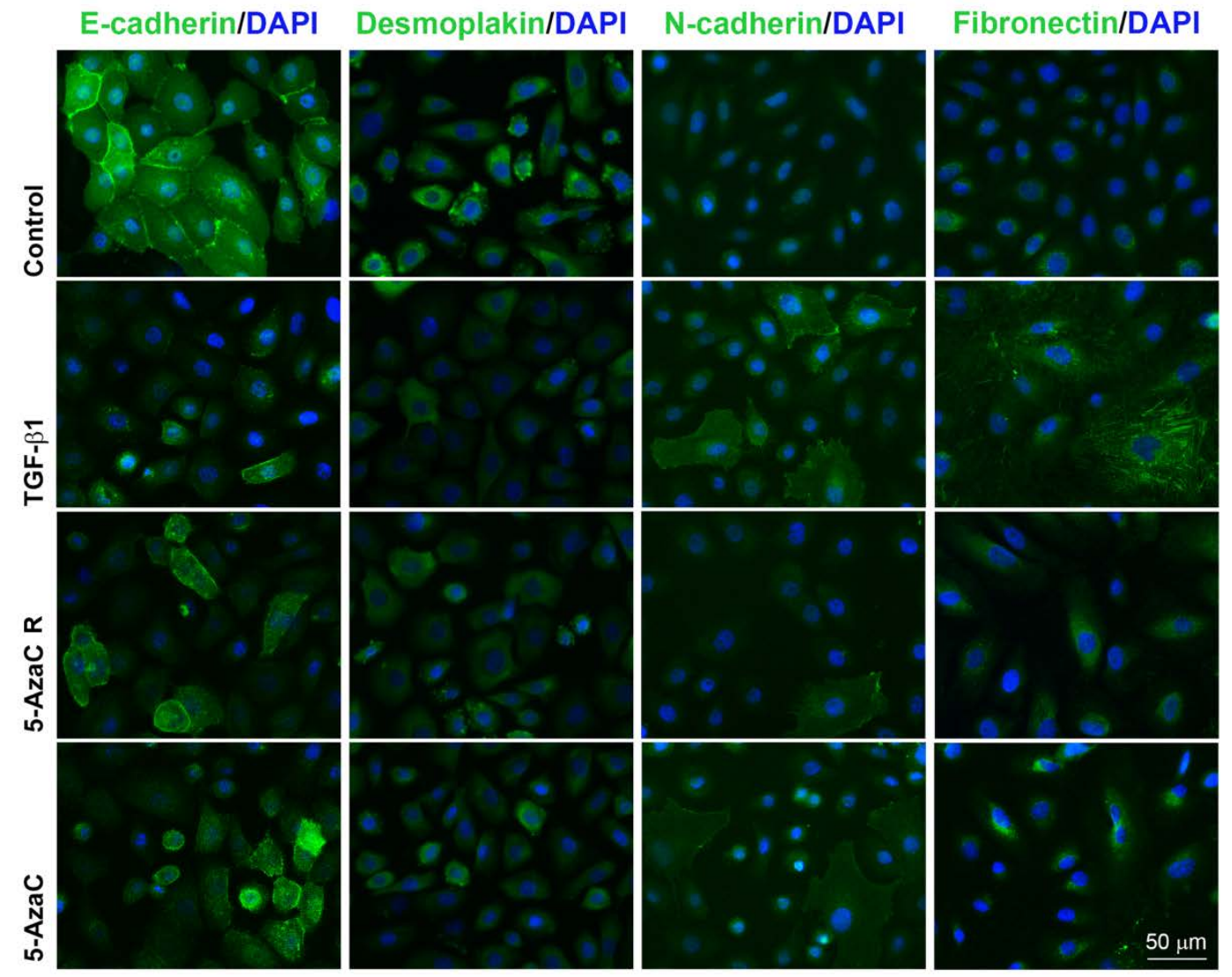

Fig. 3. The effect of the demethylating agent 5-AzaC on cell phenotype and changes in protein expression levels in TGF- $\beta 1$ treated $\mathrm{HCjE}$ cells. Morphological changes (A) and cell migration analysis (B) of $\mathrm{HCjE}$ cells in the sixth passage after 5-AzaC treatment with a recovery period (5-AzaC R) and 5-AzaC treatment without a recovery period (5-AzaC). Representative images of the in vitro wound healing assay at $0 \mathrm{~h}$ and $24 \mathrm{~h}$ are shown. The graph represent the area (in pixels) of the wound covered by the cells after $24 \mathrm{~h}$. Data are shown as the means \pm SEMs from three independent experiments, * $\mathrm{P}<0.05$ and $* *$ $\mathrm{P}<0.01$ compared to the control, \#\# $\mathrm{P}<0.01$ compared to the TGF- $\beta 1$-treated HCjE cells, $++\mathrm{P}<0.01$ compared to the 5 -AzaC R-treated HCjE cells. (C) Immunofluorescence analysis of control, TGF- $\beta 1,5-A z a C$, and 5-AzaC-treated HCjE cells in the sixth passage with antibodies against epithelial (E-cadherin and desmoplakin) and mesenchymal ( $\mathrm{N}$-cadherin and fibronectin) marker proteins (green fluorescence). Nuclei are stained with DAPI (blue fluorescence).

Both 5-AzaC treatments induced morphological changes back towards the epithelial phenotype and reduced cell mobility with respect to TGF- $\beta 1$ only-treated cells (Figs. 3A and 3B). The effects of 5- 
$\mathrm{AzaC}$ treatment with a recovery period were more pronounced than the effects of 5-AzaC treatment without a recovery period, as judged by the results of the wound healing assay (Fig. 3B) and the reduction in the number of spindle-shaped mesenchymal cells (Fig. 3A and Appendix B2).

Immunocytochemistry (ICC) confirmed that the 5-AzaC treatments were capable of restoring the epithelial phenotype to some extent (Fig. 3C). The TGF- $\beta 1$ treatment induced an almost complete loss of the epithelial markers E-cadherin and desmoplakin, while 5-AzaC treatments partially restored their expression. At the same time the expression levels of the mesenchymal markers $\mathrm{N}$ cadherin and fibronectin were markedly diminished after the 5-AzaC treatments (Fig. 3C). The powerful influence of DNA demethylation was especially observable on fibronectin expression whose level was reduced almost to control level after both 5-AzaC treatments. Based on the finding that DNA demethylating agent 5-AzaC was able to stop EMT-related changes and to partly restore epithelial phenotype in $\mathrm{HCjE}$ cells, it can be concluded that DNA methylation plays important role in EMT induction in human conjunctival epithelial cells.

\subsection{5-AzaC treatment reverses EMT by DNA demethylation of upstream regulatory factor(s)}

In order to elucidate the potential targets whose change in DNA methylation contributes to EMT induction in HCjE cells, EMT-associated gene expression profiling using the $\mathrm{RT}^{2}$ Profiler $^{\mathrm{TM}}$ PCR Array was performed. Gene expression profiling included control cells, cells treated with TGF- $\beta 1$ and cells co-treated with 5-AzaC with and without a recovery period (Fig. 4). Induction of EMT was associated with considerable alterations in gene expression; using filtering criteria for a 2-fold regulation cut-off showed that among the 84 EMT-related genes (Appendix C1), 23 genes were overexpressed and 36 were underexpressed in TGF- $\beta 1$-treated cells as compared to control cells (Fig. 4A). The 5-AzaC treatment without a recovery period induced the same outcome as TGF- $\beta 1$ treatment, while 5-AzaC treatment with a recovery period resulted in upregulation of 19 and downregulation of 38 genes when compared to control cells. Although the number of affected genes was comparable, further analysis showed that the $5-A z a C$ treatment with a recovery period induced differences in gene expression comparing to the TGF- $\beta 1$ treatment, but also comparing to the 5-AzaC treatment without a recovery period. Conversely, comparison of 5-AzaC treatment without a recovery period with the TGF- $\beta 1$ treatment revealed that only one gene was upregulated in the 5-AzaC sample (Fig. 4A).

Cluster analysis visualized in the form of a heat map confirmed that the expression profile of cells after the 5-AzaC treatment with a recovery period is closest to the control expression pattern of all treatment groups (Fig. 4B). Among genes that changed their expression after the 5-AzaC treatment with a recovery period towards the level detected in control sample are PDGFRB, CDH2, SNAI1, SNAI2, ITGA5, ZEB1, TGFB2, FN1, FOXC2, MMP2, RGS2, GEMIN2, PTP4A1, all of which are genes with a profound influence on EMT induction. 
A

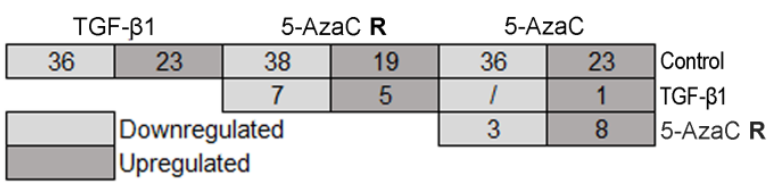

B

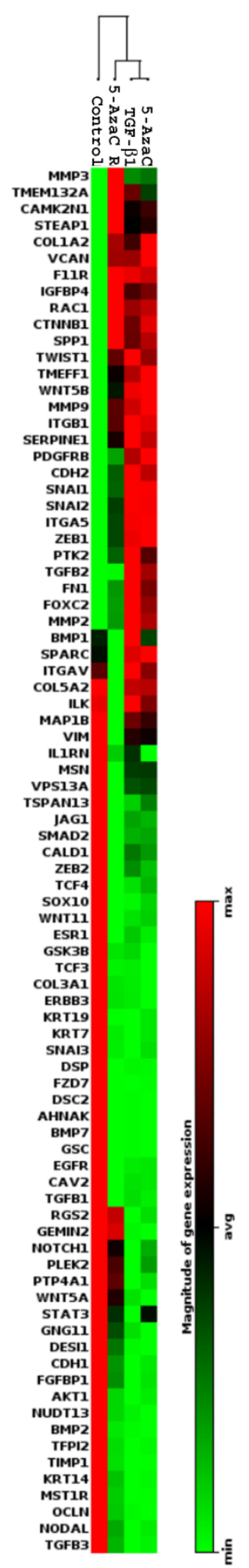

C
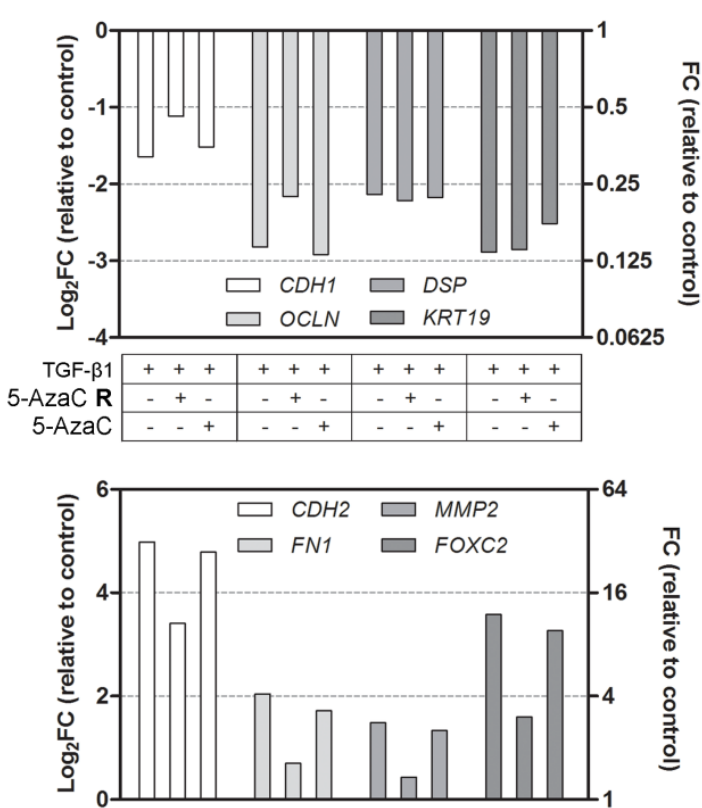

TGF- $\beta 1$ + + + $+4++\mid+++\quad+++$

5-AzaC R

$5-\mathrm{AzaC}$

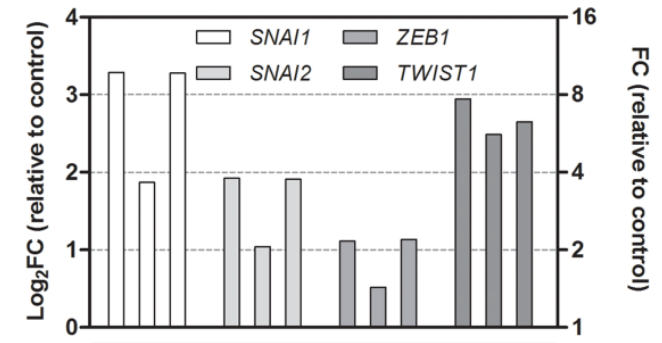

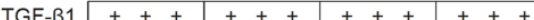

5-AzaC R

5-AzaC

D

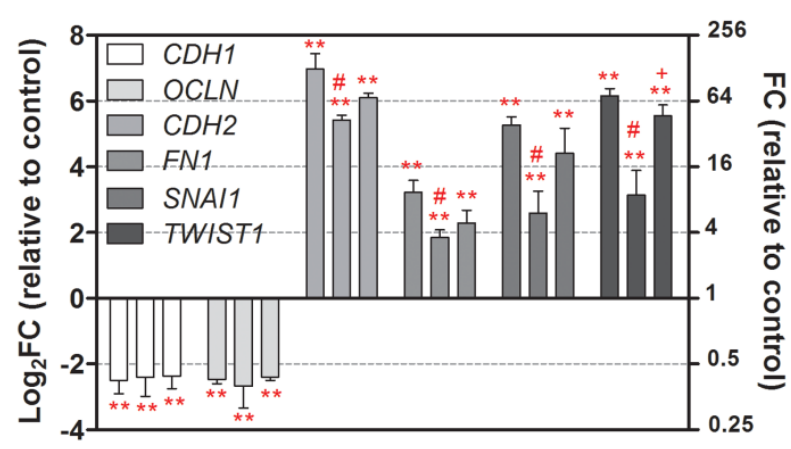

\begin{tabular}{r|c|c|c|c|c|c|}
\cline { 2 - 6 } TGF- 1 & +++ & +++ & +++ & +++ & +++ & +++ \\
\cline { 2 - 6 } 5-AzaC R & -+- & -+- & -+- & -+- & -+- & -+- \\
\cline { 2 - 6 } 5-AzaC & -++ & --+ & --+ & --+ & -++ & --+ \\
\cline { 2 - 6 } & &
\end{tabular}

Fig. 4. The effect of the demethylating agent 5-AzaC on mRNA expression of EMT-related genes in TGF- $\beta 1$-treated HCjE cells after the sixth passage. Number of differentially (2-fold regulation cut-off) expressed genes (A) and clustergram of 
magnitude of mRNA expression (B) in control, TGF- $\beta$, 5-AzaC $\mathbf{R}$ and 5-AzaC-treated HCjE cells obtained by the RT2 Profiler ${ }^{\mathrm{TM}}$ PCR Array. (C) mRNA levels of EMT-related genes in control, TGF- $\beta$, 5-AzaC $\mathbf{R}$, and 5-AzaC-treated HCjE cells as determined by the RT2 Profiler ${ }^{\mathrm{TM}}$ PCR Array. mRNA levels are relative to 5 reference genes and are expressed as log2 fold change (treated vs. control). Positive log2 fold changes indicate upregulation in treated cells as compared to the control while negative log2 fold changes indicate downregulation in treated cells as compared to the control. The equivalent fold change scale is presented on right $y$-axes. (D) Validation of several EMT marker genes in control, TGF- $\beta$, 5-AzaC R and 5-AzaC treated HCjE cells by qRT-PCR. The mRNA levels are expressed relative to GAPDH and as means of log2 fold change (treated vs. control) in three replicates \pm SEM. Positive log2 fold changes indicate upregulation in treated cells as compared to the control; negative log2 fold changes indicate downregulation in treated cells as compared to the control. The equivalent fold change scale is presented on right $y$-axes. ${ }^{*} \mathrm{P}<0.05$ and ${ }^{* *} \mathrm{P}<0.01$ compared to the control, $\# \mathrm{P}<0.05$ compared to the TGF- $\beta 1$-treated HCjE cells, $+\mathrm{P}<0.05$ compared to the 5 -AzaC R-treated HCjE cells.

Two important findings emerged after analysis of individual gene expression. First, an increase in gene expression could be expected after 5-AzaC treatment as a result of gene demethylation, but our results showed that more genes were downregulated than upregulated after both 5-AzaC treatments. Also, the affected genes were predominantly genes associated with the mesenchymal phenotype whereas genes expressed in epithelial cells, including E-cadherin, were mostly unaffected after the treatments with 5-AzaC (Fig. 4C). The most prominent alteration in gene expression was observed in $\mathrm{HCjE}$ cells treated with 5 -AzaC treatment with a recovery period, with the modulation of gene expression of EMT-related transcription factors (SNAI, ZEB, TWIST, FOXC2) and mesenchymal marker genes such as $C D H 2$ and $F N 1$ as the main consequence (Fig. 4C). When compared to TGF- $\beta 1$-treated cells, 5 -AzaC treatment with a recovery period induced a reduction in the expression of this group of genes, back towards the level observed in control cells, indicating that none of the these genes were directly affected by DNA demethylation.

The results of the EMT-related gene expression array were validated using qRT-PCR analysis that revealed that the direction of changes were consistent with the results of array analysis, with statistically significant suppression of all tested mesenchymal-specific genes in cells after 5 -AzaC treatment with a recovery period compared to TGF- $\beta 1$-treated cells (Fig. 4D). Therefore, we concluded that the main effect of the 5-AzaC treatment in reversing EMT is accomplished by DNA demethylation of upstream regulatory factor(s), instead of direct demethylation of target genes that were tested in the array.

3.4. Members of the miR-200 family are key players in the maintenance of the epithelial phenotype of $\mathrm{HCjE}$ cells

3.4.1. Changes in the profile of miRNA expression during EMT and after 5-AzaC treatments

The expression profiles in cells that underwent EMT and in cells subjected to DNA demethylation suggested that regulatory factors we are searching for had to be negative regulators of gene expression. miRNAs are the most powerful class of gene repressors because of their number and specificity of the mechanism of action. Therefore, our next aim was to obtain the expression profile of selected miRNAs in control conditions, after EMT induction, as well as after both 5-AzaC treatments, with and without a recovery period using the miRCURY LNA ${ }^{\mathrm{TM}}$ miRNA Human Cancer Focus PCR Panel (Fig. 5 and Appendix C2). Utilizing the filtering criteria of a 2-fold regulation difference in expression, 16 out of 84 miRNAs were found to be overexpressed and 11 miRNAs were repressed in TGF- $\beta 1$-treated cells in comparison with control cells (Fig. 5A). The 5-AzaC treatment with a recovery period induced overexpression of 18 and downregulation of 12 miRNAs when compared to the control level of miRNA expression. The 5-AzaC treatment without a recovery period 
induced upregulation of 16 and downregulation of 12 miRNAs as compared to the control. Among the overexpressed miRNAs, for miR-10b-5p, miR-143-3p, miR-145-5p, miR-146a-5p, miR-196a-5p and miR-10a-5p, a greater than 10-fold increase in expression was detected (Fig. 5B). All listed miRNAs, except miR-10b-5p and miR-10a-5p, displayed increased expression to a certain extent after some of the 5-AzaC treatments, suggesting possible involvement of DNA methylation in the regulation of their expression.

A

\begin{tabular}{|c|c|c|c|c|c|c|}
\hline \multicolumn{2}{|c|}{ TGF- $\beta 1$} & \multicolumn{2}{|c|}{ 5-AzaC R } & \multicolumn{2}{|c|}{ 5-AzaC } & \\
\hline 11 & 16 & 12 & 18 & 12 & 16 & Control \\
\hline & & 1 & 2 & 4 & 1 & TGF- $\beta 1$ \\
\hline & wh & ted & & 5 & 1 & 5-AzaC R \\
\hline
\end{tabular}

B

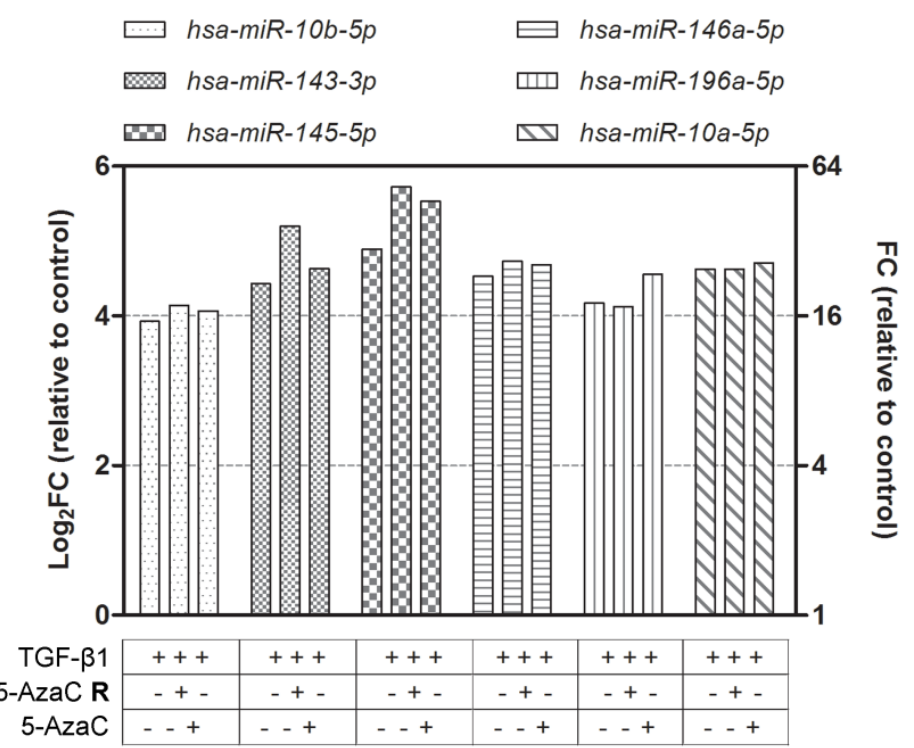

C
hsa-miR-200a-3p
$\Delta$ hsa-miR-200b-3p
hsa-miR-141-3p
ש hsa-miR-200c-3p
Wsa-miR-182-5p

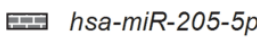
سU hsa-miR-148a-3p
hsa-miR-20b-5p
$\succeq$ hsa-miR-18a-5p
Wsa-miR-215-5p

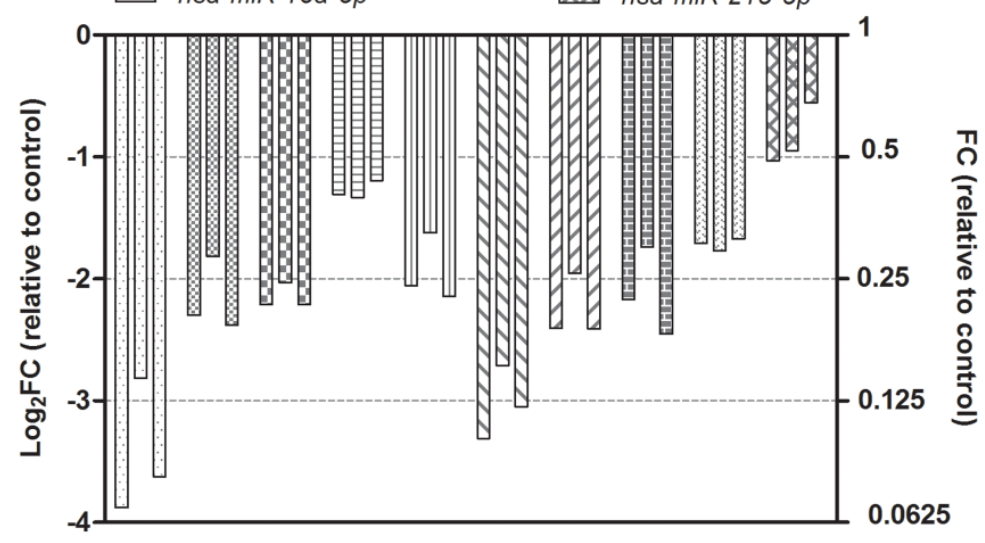

TGF- 31 1

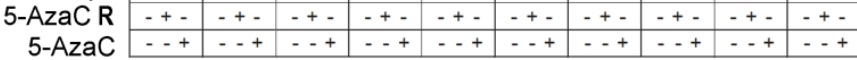


Fig. 5. The effect of the demethylating agent 5-AzaC on the expression of EMT-related miRNA in TGF- $\beta 1$-treated HCjE cells after the sixth passage estimated using the miRCURY LNA miRNA Cancer Focus PCR Panel. (A) Number of differentially (2fold regulation cut-off) expressed miRNAs between control, TGF- $\beta$, 5-AzaC R and 5-AzaC-treated HCjE cells. Expression levels of upregulated (B) and downregulated (C) EMT-related miRNAs in control, TGF- $\beta$, 5-AzaC R and 5-AzaC-treated HCjE cells. The mRNA levels are expressed relative to 10 reference miRNA as log2 fold change (treated vs. control). Positive log2 fold changes indicate upregulation in treated cells as compared to the control; negative log 2 fold changes indicate downregulation in treated cells as compared to the control. The equivalent fold change scale is presented on right $y$-axes.

Among the miRNAs that were downregulated after EMT induction, the most pronounced change in expression was detected for members of the miR-200 family of miRNAs, miR-200a, -200b, -141 and 200c (Fig. 5C). In addition to them, miR-182, -18a, -148a, -205, -20b and -215 were downregulated as a result of TGF- $\beta 1$ treatment. The treatment with 5-AzaC with a recovery period shifted the expression of miR-200 family, miR-18a and miR-205 towards the control level. Thereby the 5-AzaC treatment with a recovery period induced a 2 -fold increase in expression of miR-200a with respect to TGF- $\beta 1$-only treated cells. At the same time, the 5 -AzaC treatment without a recovery period had a negligible effect on the expression levels of the tested miRNAs. Influence on miR-200 family expression level could explain the stronger impact of the 5-AzaC treatment with a recovery period on EMT regression as observed throughout the study.

3.4.2. Alteration of DNA methylation status of the promoters of the miR-200 family during EMT and after the 5-AzaC treatment with a recovery period

In order to examine the possibility that DNA methylation effects miR-200 expression levels in $\mathrm{HCjE}$ cells, the DNA methylation status of both miR-200 loci (Figs. 6A and 7A) was assessed in control HCjE cells, HCjE cells after induction of EMT and in cells exposed to the 5-AzaC treatment with a recovery period. DNA methylation was analyzed by methylation-sensitive high resolution melting (HRM) (Figs. $6 \mathrm{~B}$ and $7 \mathrm{~B}$ ) and bisulfite sequencing (Bis-Seq) (Figs. $6 \mathrm{C}$ and $7 \mathrm{C}$ ). The region selected for DNA methylation analysis of the miR-200a/b/429 cluster is localized within the CpG island and contains the promoter sequence and transcription start site (TSS), while for methylation status analysis of the miR-200c/141 cluster, sequence upstream of TSS was chosen (Figs. 6A and 7A). HRM analysis of the miR-200a/b/429 cluster covered the region from -41 to +208 , while region subjected to Bis-Seq encompassed sequences from -118 to +208 with regard to the position of TSS marked as +1 . According to HRM analysis, the prolonged treatment with TGF- $\beta 1$ led to a statistically significant 2.5fold increase in the percentage of DNA methylation of the selected region of miR-200a/b/429 cluster as compared to the control state (Fig. 6B). Co-treatment with 5-AzaC induced a statistically significant reduction in the level of DNA methylation in comparison to the TGF- $\beta 1$ treatment which declined almost to control level. Bis-Seq covered a broader region for analysis and the results revealed that the treatment with TGF- $\beta 1$ induced an overall increase in the level of DNA methylation (Fig. 6C). The entire region downstream from TSS was found to be markedly methylated, while in the upstream region only two CpGs (at positions -44 and -81) were changed. The analyzed region was targeted by the 5-AzaC treatment, as CpGs that were methylated in the TGF- $\beta 1$ samples, converted status to hemimethylated or unmethylated after the treatment with 5-AzaC (Fig. 6C).

The same set of primers was used for HRM and Bis-Seq of the selected region of the miR-200c/141 cluster, which covered the sequences from -76 to -264 relative to TSS (Fig. 7A). HRM analysis revealed a statistically significant and substantial (more than 4-fold) increase in the percentage of 
CpG methylation as a result of the treatment with TGF- $\beta 1$ (Fig. 7B). Treatment with 5-AzaC diminished DNA methylation level to a degree that was statistically different from both, control and TGF- $\beta 1$-treated levels. Bis-Seq basically confirmed the previous findings as almost all analyzed CpGs were subjected to change in methylation status: towards an increase in DNA methylation after the TGF- $\beta 1$ treatment, and in the opposite direction after the 5-AzaC treatment (Fig. 7C).

A

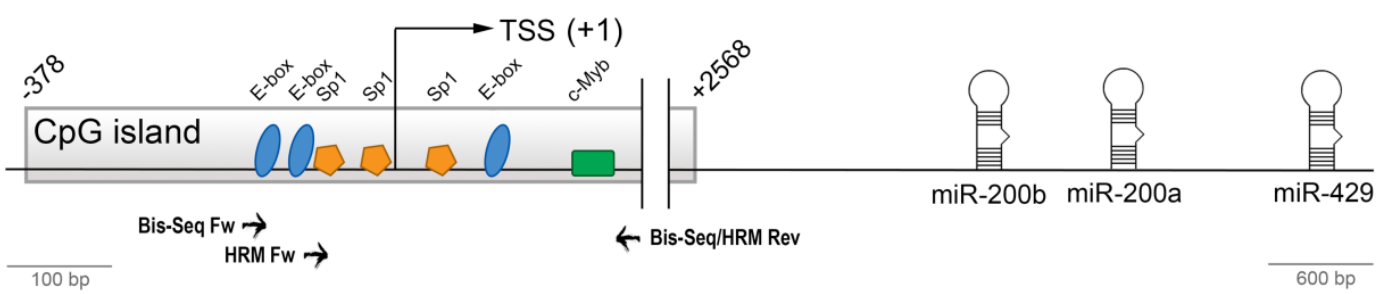

B
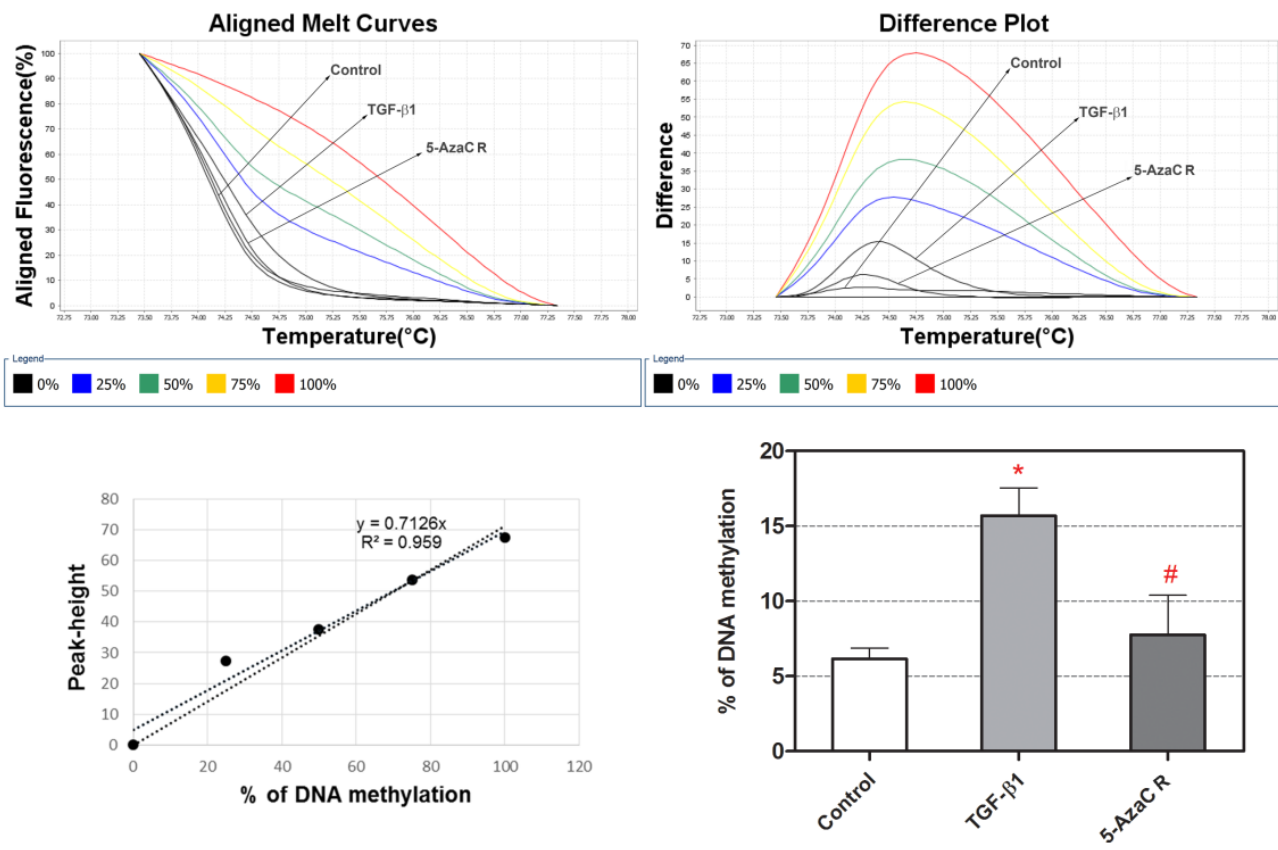

C

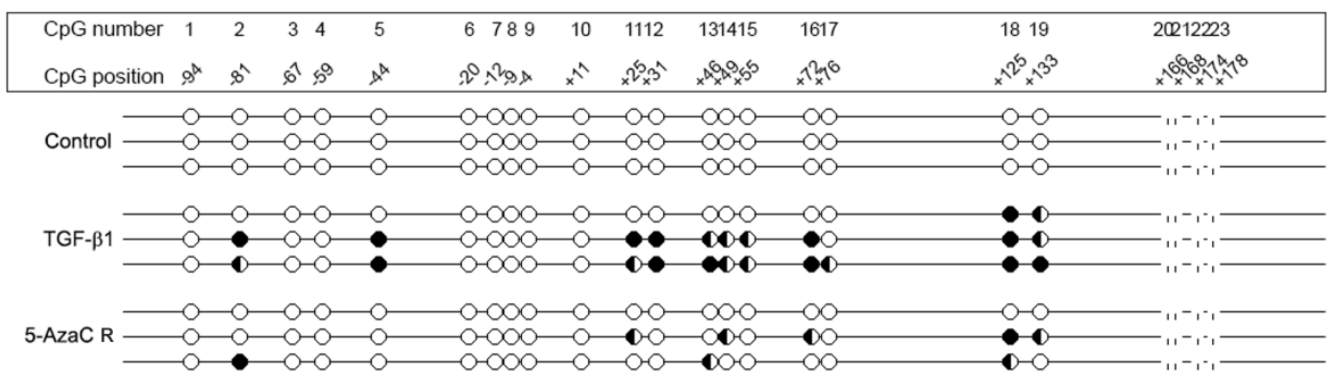

Fig. 6. The influence of the $5-\mathrm{AzaC}$ treatment with a recovery period on the DNA methylation status of the miR-200b/a/429 cluster in TGF-B1-treated HCjE cells after the sixth passage. (A) Schematic representation of miR-200b/a/429 genomic loci. $\mathrm{CpG}$ island, transcription start site (+1), putative transcription factors binding sites and positions of primers used for HRM and Bis-Seq are marked. (B) DNA methylation levels of the selected region obtained by HRM analysis in control, TGF- $\beta$, and 5-AzaC R-treated HCjE cells. Representative aligned melt curves and difference plots showing positions of analyzed curves 
with respect to $0,25,50,75$, and $100 \%$ methylated standards. The results on the graph represent the percentage of DNA methylation obtained from standard curves for three replicates and are expressed as the means \pm SEMs; ${ }^{*} \mathrm{P}<0.05$ compared to the control, \# P<0.05 compared to the TGF- $\beta 1$-treated HCjE cells. (C) Bisulfite genomic sequencing analysis of the miR200b/a/429 CpG island in control, TGF- $\beta$ and 5-AzaC R-treated HCjE cells presented in triplicate. The analyzed CpGs are numbered 1 to 23 and their positions with regard to the TSS are indicated. The presence of unmethylated or methylated CpGs is marked by white or black circles, respectively.

A

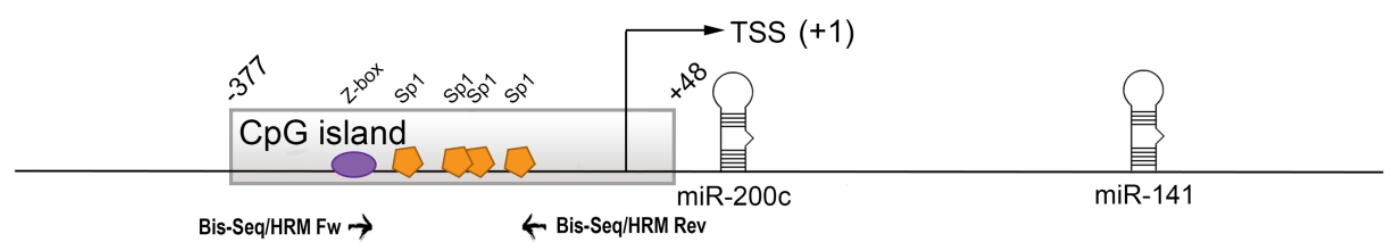

B
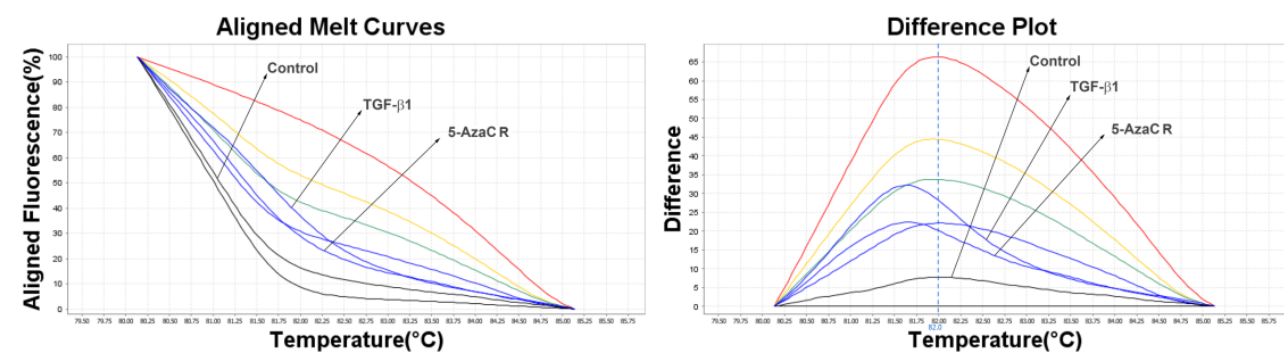

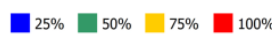
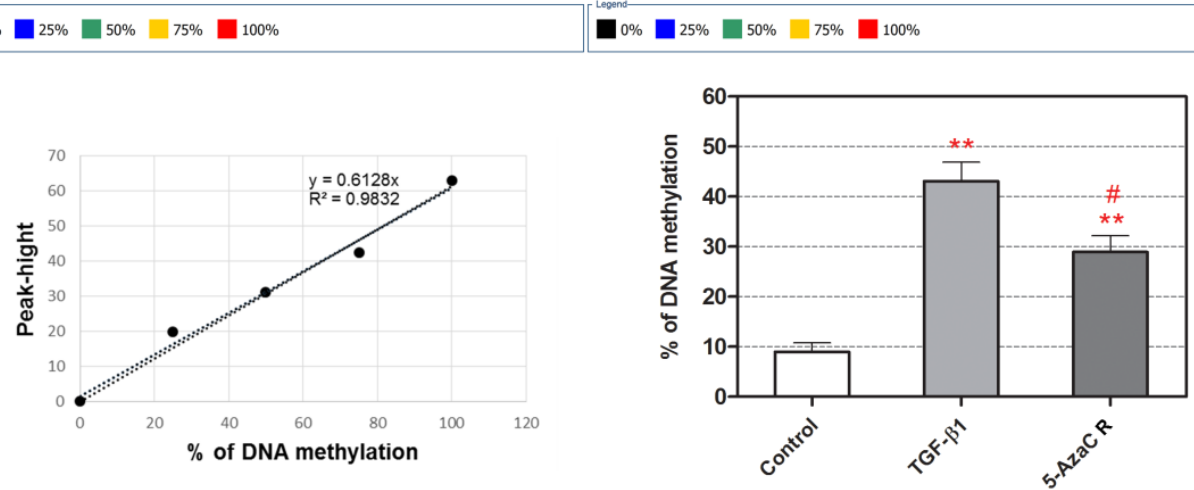

C

CpG number position

Fig 7. The influence of the $5-A z a C$ treatment with a recovery period on the DNA methylation status of the miR-200c/141 cluster in TGF- $\beta 1$-treated HCjE cells after the sixth passage. (A) Schematic representation of miR-200c/141 genomic loci. $\mathrm{CpG}$ island, transcription start site (+1), putative transcription factors binding sites and positions of primers used for HRM and Bis-Seq are marked. (B) DNA methylation levels of the selected region obtained by HRM analysis in control, TGF- $\beta$, and 5-AzaC R-treated HCjE cells. Representative aligned melt curves and difference plots showing positions of analyzed curves with respect to $0,25,50,75$, and $100 \%$ methylated standards. The results on the graph are presented as the percentage of DNA methylation obtained from standard curves for three replicates and are expressed as means \pm SEMs; ${ }^{* *} \mathrm{P}<0.01$ 
compared to the control, \# $\mathrm{P}<0.05$ compared to the TGF- $\beta 1$-treated HCjE cells. (C) Bisulfite genomic sequencing analysis of the miR-200c/141 CpG island in control, TGF- $\beta$, and 5-AzaC R-treated cells presented in triplicate. The analyzed CpGs are numbered 1 to 11 and their positions with regard to the TSS are indicated. The presence of unmethylated or methylated $\mathrm{CpGs}$ is marked by white or black circles, respectively.

\section{Discussion}

Infections, inflammations, and mechanical stresses of conjunctiva could result in conjunctival fibrosis, which, if severe, may impair vision (Friedlander, 2007). EMT emerged as an inevitable part of every fibrotic process and numerous findings indicate its important role in the fibrosis-based eye conditions. Therefore, targeting EMT could be suitable therapeutic approach for fibrosis-related eye diseases, but target genes specific for particular cell type or condition are still undefined (He et al., 2013). This study aimed to unveil multilayered regulatory mechanisms that trigger EMT in human conjunctival epithelial (HCjE) cells.

TGF- $\beta$ proteins are considered to be major factors involved in the induction of EMT, as they trigger downstream signaling pathways that ultimately results in the activation of the EMT-related transcription factors (Miyazono, 2009). Although all three TGF- $\beta$ isoforms are produced by ocular epithelial cells (Saika et al., 2009), potential of particular TGF- $\beta$ isoform to induce EMT in conjunctival cells was unknown. We found that prolonged treatment of HCjE cells with TGF- $\beta 1$ had higher potential for EMT induction comparing to TGF- $\beta 2$ or even their combination. Although TGF- $\beta 2$ has been considered as the key TGF- $\beta$ isoform for ocular physiology and the prevalent isoform in aqueous humor (Saika et al., 2001), our results indicate that TGF- $\beta 2$ was ineffective in inducing EMTrelated transcription factors ZEB2 and TWIST1. As a consequence, TGF- $\beta 2$ treated cells demonstrated lower mobility and less pronounced morphological changes than $\mathrm{HCjE}$ cells treated with TGF- $\beta 1$. The importance of TGF- $\beta 2$ for conjunctival scarring processes was proposed and tested using neutralizing monoclonal antibodies against TGF- $\beta 2$. TGF- $\beta 2$ neutralization has been shown to inhibit proliferation and migration of Tenon's capsule fibroblasts in vitro and to reduce subconjunctival scarring after glaucoma filtration surgery in the rabbit (Cordeiro et al., 1999). Although one small clinical study provided promising initial results (Siriwardena et al., 2002), a larger randomized controlled trial failed to show a difference in the success of trabeculotomy after TGF- $\beta 2$ neutralization when compared with the control group (Khaw et al., 2007). Some authors hypothesized that targeting TGF- $\beta 1$ simultaneously could overcome the failure of the TGF- $\beta 2$ neutralization alone to suppress conjunctival scarring (Saika et al., 2009; Zada et al., 2018). Besides, the importance of EMT might also be underestimated in the above-mentioned studies, and along with this, the observed high potential to induce EMT suggests that TGF- $\beta 1$ could be a major player in conjunctival fibrosis.

Aberrant DNA methylation has been shown to accompany EMT-related ocular diseases such as pterygium, as well as age-related and secondary cataract (Riau et al., 2011; Wang and Guan, 2017). DNA demethylation emerged as an efficient approach for EMT cessation in different cancer studies (Chen et al., 2004; Chen et al., 2013; Jiang et al., 2016), although ineffective in certain cell lines and even capable of inducing EMT in prostate cancer cells (Lee et al., 2016). Dual nature of DNA demethylation was also evident from studies of ocular fibrotic conditions. It has been shown that 5- 
aza-2'-deoxycytidine upregulated the expression of pro-fibrotic proteins and induced the differentiation of human Tenon's capsule fibroblasts (Fu et al., 2017). On the other hand, zebularine, another DNA demethylating agent, was capable of inhibiting attachment, migration and proliferation (Zhou et al., 2012) and of suppressing TGF- $\beta$-induced EMT in lens epithelial cells (Zhou et al., 2011). We found that 5-AzaC was able to stop EMT-related morphological changes and to reduce ability of $\mathrm{HCjE}$ to migrate, indicating importance of DNA methylation in EMT process. Thereby, treatment with 5-AzaC with recovery period was more effective than 5-AzaC treatment without recovery, suggesting that stronger impact of DNA demethylation can be seen after multiple cell divisions or/and after a certain period of time. The same was evident after profiling of EMT-associated gene expression which underlined that treatment with 5-AzaC with recovery period induced decrease in expression of mesenchymal marker genes in comparison with TGF- $\beta 1$-treated cells, as a major effect. This was accompanied with only slight increase in expression of epithelial marker genes, E-cadherin (CDH1) and occludin (OCLN). The latter is interesting as hypermethylation of the E-cadherin promoter was shown to be an important mechanisms in the loss of E-cadherin gene expression during cancer progression (Berx and van Roy, 2009). The treatment with 5-AzaC was capable of restoring Ecadherin expression, epithelial cell morphology and of reducing metastasis in 11 different cancer cell lines (Nam et al., 2004; Yoshiura et al., 1995). Besides, it has been suggested that the hypermethylation of the E-cadherin promoter is involved in reducing the expression of E-cadherin protein and thereby in the development of pterygium and during $C$. trachomatis infection (Rajic et al., 2017; Young et al., 2010). Although the treatment with 5-AzaC with recovery period induced some increase in $\mathrm{CDH} 1$ expression, the observed change in E-cadherin mRNA expression could not be responsible for stopping and partially reversing EMT. The selective effect of 5-AzaC treatment, detected as a diminishment of mesenchymal marker genes expression, suggested that none of the examined marker genes is direct target of 5-AzaC. The observed effects were ascribed to DNA demethylation of miRNAs, well described class of transcriptional repressors.

Although the key role of miRNAs in regulation of EMT is already established in various model systems (Abba et al., 2016; Exposito-Villen et al., 2018), data on the EMT-related change in miRNA pattern in conjunctival cells are still missing. The aberrant expression of different miRNAs has been correlated with different eye disorders (Raghunath and Perumal, 2015) and conjunctiva inflammation after $C$. trachomatis infection (Derrick et al., 2017). Here, we examined the expression profile of selected miRNAs in HCjE cells after EMT induction, but also after 5-AzaC treatments with and without a recovery period that allowed us to identify particular miRNAs which are, at the same time, involved in EMT induction and regulated by DNA methylation. Among overexpressed miRNAs in HCjE cells that undergone EMT, miR-10b-5p, miR-143-3p, miR-145-5p and miR-10a-5p are under direct transcriptional control of TGF- $\beta$ (Liu et al., 2013; Long and Miano, 2011), which can explain the strong induction of their expression in HCjE cells treated with TGF- $\beta 1$. miR10a and miR10b have been identified as important regulators of EMT, capable of promoting cell migration and invasion in various cancer cells (Han et al., 2014; Yan et al., 2015; Zhang et al., 2015). In line with that, we observed more than 10 fold increase in expression of miR10a and miR10b in HCjE cells than undergone EMT. Besides, neither of 5-AzaC treatments applied in this study did not impact expression of those miRNAs, suggesting that other mechanisms than DNA methylation are involved in miR10a and miR10b expression. The precise role of miR-143 and miR-145 in ocular tissue is not 
defined yet. Examination of miR-145 level in pterygium tissue from 253 patients revealed a negative correlation between pterygium severity and the level of miR-145 expression, which is in accordance with its proposed tumor suppressor activity (Chien et al., 2013). Still, the expression levels of miR143 and miR-145 in primary pterygia have been shown to be significantly higher in comparison with the levels detected in control conjunctiva, indicating that the expression of the miR-143/145 cluster might be triggered in pathologic conditions (Teng et al., 2018). In accordance, we found that the induction of EMT in HCjE cells was associated with upregulation of the miR-143/145 cluster. Treatments with 5-AzaC, especially the treatment with 5-AzaC with recovery period, induced a further increase in their expression, confirming the previous findings that changes in DNA methylation of miR-143 and miR-145 promoter regions correlate with their levels of expression which was documented in several carcinomas and in vascular smooth muscle cells (Gao et al., 2019; Xia et al., 2015; Zhang et al., 2016). The effects of 5-AzaC treatment represent total sum of outcomes resulting from the suppression and activation of numerous genes which are regulated by DNA methylation. After induction of EMT in HCjE cells, the net effect of DNA demethylation was cessation of EMT process, despite the fact that with respect to miR-143 and miR-145, the 5-AzaC treatment most probably shifted the equilibrium towards EMT.

Among the miRNAs that were downregulated in $\mathrm{HCjE}$ cells after EMT induction, the most pronounced reduction was found in the expression levels of the miR-200 family members. The miR200 family of miRNAs consists of five members organized in two clusters located on chromosome 1 (miR-200a, -200b and -429) and chromosome 12 (miR-200c and -141). Cancer studies revealed that the miR-200 family can be considered as the guardian of the epithelial phenotype and one of the key regulators of EMT, involved in its suppression (Bracken et al., 2008; Burk et al., 2008; Gregory et al., 2008b). Examination of miRNA expression profiles in 60 cell lines and in ovarian cancer patients has identified the miR-200 family of miRNAs as a common marker for E-cadherin-positive and vimentinnegative cancer cells (Park et al., 2008). The miR-200 family supports the epithelial phenotype through direct targeting the mRNAs of EMT-related transcription factors ZEB1 and ZEB2. Multiple target sites for miR-200a, -141, miR-200b, -200c and miR-205 in the 3'UTR of ZEB1 and ZEB2 mRNA have been identified and it was shown that these miRNAs cooperatively regulate their expression, thus critically influencing the potential for metastasis and tumor progression (Gregory et al., 2008a). Also, pterygium is associated with significant changes in expression of multiple miRNAs, thereby members of the miR-200 family, miR-200a, miR-200b and miR-141 were found downregulated in pterygium samples when compared to normal conjunctiva (Engelsvold et al., 2013). An important role of miR-200a in EMT and pterygium induction, through upregulation of ZEB1, ZEB2 and SNAIL gene expression was shown on primary pterygium cells (Wu et al., 2016). It is not surprising that common mechanisms lie behind EMT-based conditions such as pterygium and cancer metastasis, since pterygium has been considered a condition characterized by noncancerous but uncontrolled cell proliferation originating from the conjunctiva (Chui et al., 2011; Tsai et al., 2005). Here we showed that EMT induced by TGF- $\beta 1$ in $\mathrm{HCjE}$ cells that have not undergone malignant transformation follow the same pattern of miR-200 family downregulation as observed in carcinoma or pterygium. Aside from the miR-200 family, numerous other miRNAs have been described as critically important for EMT induction (Abba et al., 2016; Exposito-Villen et al., 2018). However, our results suggest that the miR-200 family, in particular miR-200a and miR-200b, are fundamental 
protagonists that maintain the epithelial phenotype of human conjunctival cells, as their downregulation correlates with the transition to the mesenchymal state.

The expression of the miR-200 family is regulated on multiple levels. ZEB1 was shown to be repressed by miR-200c and at the same time ZEB1 directly suppresses transcription of miR-141 and 200c by binding to E-box sites in their promoter region (Burk et al., 2008). It was also demonstrated that ZEB1 and ZEB2 repress miR-200b, -200a and -429, suggesting double-negative feedback loop between the miR-200 family and ZEB factors to be an important EMT regulator (Bracken et al., 2008). An additional level of regulation of the expression of the miR-200 family is mediated by DNA methylation. Screening a panel of cancer cell lines, representing the most common human tumor types, revealed that promoter DNA methylation of both miR-200 loci is in correlation with miR-200 expression levels and that it corresponds to the epithelial/mesenchymal state (Davalos et al., 2012). Likewise, in normal colon mucosa, epithelial cells from the colorectal crypts had unmethylated CpG islands in both miR-200 loci and expressed corresponding transcripts, while lamina propria cells did not express these miRNAs, together with hypermethylation of their promoters (Davalos et al., 2012). In line with that, we found that treatment of $\mathrm{HCjE}$ cells with 5-AzaC with a recovery period moved the expression of miR-200 family towards the control level, confirming involvement of DNA methylation in their expression in conjunctival cells. HRM and Bis-Seq analysis revealed that TGF- $\beta$ induced EMT was accompanied by increase in CpG methylation of promoters of both miR-200 loci. Besides, we showed that the analyzed region was targeted by the 5-AzaC treatment, as analyzed CpGs changed status towards decrease in DNA methylation after the 5-AzaC treatment. It can be concluded that the expression level of both miR-200 loci correlates with DNA methylation status of their promoters and is associated with epithelial or mesenchymal state of HCjE cells.

The functional implication of these alterations in $\mathrm{CpG}$ methylation status might be modification of binding abilities of transcription factors to differently methylated DNA. Prediction of transcription factor binding sites using Match ${ }^{\mathrm{TM}}$ revealed that the selected region of miR-200a/b/429 contain, among others, three SP1 and one C-MYB binding site and three ZEB binding sites already known from the literature (Bracken et al., 2008; Burk et al., 2008) (Fig. 6A), while the analyzed sequence of the miR-200c/141 cluster contains one ZEB and four SP1 binding sites (Fig. 7A). ZEBs are well-known repressors, whereas SP1 and c-MYB act as activators of the expression of miR-200 family (Kolesnikoff et al., 2014; Pieraccioli et al., 2013). It has been shown that the net result of this activator/repressor interplay depends on the presence of the transcriptional repressor ZEB. Namely, SP1 and c-MYB were shown to drive the expression of miR-200 family members and to maintain the epithelial phenotype in that way. The appearance of ZEB proteins after EMT induction prevents the binding of activators so that repression prevails over activation (Kolesnikoff et al., 2014; Pieraccioli et al., 2013). Taking into account that some of the differently methylated $\mathrm{CpGs}$ in the promoter regions of the miR-200 family that were analyzed in this study belong to SP1 and c-MYB binding sites, it can be proposed that increased CpG methylation in TGF- $\beta 1$-treated $\mathrm{HCjE}$ cells disrupts the binding of activators and together with the simultaneous appearance of ZEB repressors, consequently results in downregulation of miR-200 members, which in turn allow transition to the mesenchymal state.

Our results indicate that the methylation status of both miR-200 loci correlates with their expression level in $\mathrm{HCjE}$ cells, and that it could be a decisive mechanism in determination of cell fate in the 
context of initiating the EMT process. In our experimental setup, the immediate cause of observed change in the methylation pattern of miR-200 loci during EMT could be the result of the treatment with TGF- $\beta 1$. Previous studies showed a wide range of effects of the TGF- $\beta 1$ treatment on DNA methylation profiles, depending on the cell type and experimental model, from the absence of any detected changes (McDonald et al., 2011) to reversible hypermethylation (Cardenas et al., 2014; Carmona et al., 2014). However, it seems that TGF- $\beta 1$ is capable of inducing CpG methylation and that this ability depends on treatment duration. Namely, Gregory et al. showed that DNA methylation of both miR-200 loci progressively increased with the duration of TGF- $\beta 1$ exposure and found a correlation between the boost in DNA methylation and reduced miR-200 expression (Gregory et al., 2011), which is in accordance with results that we obtained. Increased expression and activity of DNMTs has been linked with hypermethylation of miR-200 loci and EMT induction (Luo et al., 2019; Pistore et al., 2017). Which DNMT enzyme is involved in the hypermethylation of miR-200 loci in HCjE cells is the aim of future research.

\section{Conclusions}

The aim of this study was to identify the key player(s) involved in the induction of EMT in HCjE cells which could serve as potential specific targets in the treatment of EMT-related conjunctival diseases. We found that HCjE cells are prone to EMT induction after prolonged treatment with TGF- $\beta 1$, suggesting, as an in vivo implication, potential of conjunctival cells to enter EMT under conditions of chronic inflammation. This study provides additional understanding of the epigenetic regulation of the process of EMT, in particular unveiling the importance of DNA methylation. Treatment of HCjE cells with the demethylating agent 5-AzaC revealed that DNA methylation assumes central place in the promotion of EMT process. Importantly, the results of our experiments showed a higher impact of the 5-AzaC treatment after a time delay than immediately after the treatment on the cessation or reversion of EMT. Our results also point to the highly selective and specific effects of 5-AzaC with the most pronounced influence on the expression of miRNAs. Finally, we established that the miR-200 family, especially miR-200a and miR-200b, are crucial players in EMT induction in HCjE cells. Identification of the miR-200 family as targets for potential therapeutic treatment is of particular importance since lack of target gene specificity is considered as a major problem in the application of epigenetic agents for ocular diseases (He et al., 2013). The epigenetic field is constantly increasing and improvements in technology already allow targeted DNA demethylation using a CRISPRs/Cas9 based epigenetic tool (Choudhury et al., 2016), while accessibility of the eye enables local application of robust gene editing tools. As members of the miR-200 family are organized in clusters, targeted demethylation of a single promoter will induce expression of all cluster members. Therefore, taking all mentioned benefits into account, we suggest directed DNA demethylation of the miR-200 family as a novel therapeutic strategy in the treatment of conjunctival conditions associated with EMT.

\section{Author contributions}

Conception and design of the experiments: NG, MV, AIK, TBA; Performed the cell culture experiments: JR, MĐ, SD; Performed the RTqPCR experiments: JR, MĐ, JAJ; Performed the DNA methylation experiments: JR, AT, AU; Interpretation of data and preparation of figures: JR, NG, MM; 
Wrote the paper: NG, JR; Critical revision of the manuscript: MV, GP, AIK, TBA. All authors approved the final version for publication.

Declarations of interest: none

\section{Acknowledgements}

This work was supported by the Ministry of Science of the Republic of Serbia (Grant No. 173020); "Laura Bassi Centers of Expertise" program of the Austrian Federal Ministry of Economy through the Austrian Research Promotion Agency (FFG project number 822768).

\section{REFERENCES:}

Abba, M.L., Patil, N., Leupold, J.H., Allgayer, H., 2016. MicroRNA Regulation of Epithelial to Mesenchymal Transition. J Clin Med 5.

Agrawal, K., Das, V., Vyas, P., Hajduch, M., 2018. Nucleosidic DNA demethylating epigenetic drugs - A comprehensive review from discovery to clinic. Pharmacol Ther 188, 45-79.

Berx, G., van Roy, F., 2009. Involvement of members of the cadherin superfamily in cancer. Cold Spring Harb Perspect Biol 1, a003129.

Bracken, C.P., Gregory, P.A., Kolesnikoff, N., Bert, A.G., Wang, J., Shannon, M.F., Goodall, G.J., 2008.

A double-negative feedback loop between ZEB1-SIP1 and the microRNA-200 family regulates epithelial-mesenchymal transition. Cancer Res 68, 7846-7854.

Burk, U., Schubert, J., Wellner, U., Schmalhofer, O., Vincan, E., Spaderna, S., Brabletz, T., 2008. A reciprocal repression between ZEB1 and members of the miR-200 family promotes EMT and invasion in cancer cells. Embo Rep 9, 582-589.

Cardenas, H., Vieth, E., Lee, J., Segar, M., Liu, Y.L., Nephew, K.P., Matei, D., 2014. TGF-beta induces global changes in DNA methylation during the epithelial-to-mesenchymal transition in ovarian cancer cells. Epigenetics-Us 9, 1461-1472.

Carmona, F.J., Davalos, V., Vidal, E., Gomez, A., Heyn, H., Hashimoto, Y., Vizoso, M., Martinez-Cardus, A., Sayols, S., Ferreira, H.J., Sanchez-Mut, J.V., Moran, S., Margel, M., Castella, E., Berdasco, M., Stefansson, O.A., Eyfjord, J.E., Gonzalez-Suarez, E., Dopazo, J., Orozco, M., Gut, I.G., Esteller, M., 2014. A Comprehensive DNA Methylation Profile of Epithelial-to-Mesenchymal Transition. Cancer Res 74, 5608-5619.

Chen, Q., Lipkina, G., Song, Q., Kramer, R.H., 2004. Promoter methylation regulates cadherin switching in squamous cell carcinoma. Biochem Biophys Res Commun 315, 850-856.

Chen, Y., Wang, K., Leach, R., 2013. 5-Aza-dC treatment induces mesenchymal-to-epithelial transition in 1st trimester trophoblast cell line HTR8/SVneo. Biochem Biophys Res Commun 432, 116-122.

Chien, K.H., Chen, S.J., Liu, J.H., Woung, L.C., Chen, J.T., Liang, C.M., Chiou, S.H., Tsai, C.Y., Cheng, C.K., Hu, C.C., Peng, C.H., 2013. Correlation of MicroRNA-145 Levels and Clinical Severity of Pterygia. Ocul Surf 11, 133-138.

Choi, S.K., Pandiyan, K., Eun, J.W., Yang, X., Hong, S.H., Nam, S.W., Jones, P.A., Liang, G., You, J.S., 2017. Epigenetic landscape change analysis during human EMT sheds light on a key EMT mediator TRIM29. Oncotarget 8, 98322-98335.

Choudhury, S.R., Cui, Y., Lubecka, K., Stefanska, B., Irudayaraj, J., 2016. CRISPR-dCas9 mediated TET1 targeting for selective DNA demethylation at BRCA1 promoter. Oncotarget 7, 46545-46556.

Chui, J., Coroneo, M.T., Tat, L.T., Crouch, R., Wakefield, D., Di Girolamo, N., 2011. Ophthalmic Pterygium A Stem Cell Disorder with Premalignant Features. American Journal of Pathology 178, 817-827. 
Cordeiro, M.F., Gay, J.A., Khaw, P.T., 1999. Human anti-transforming growth factor-beta2 antibody: a new glaucoma anti-scarring agent. Invest Ophthalmol Vis Sci 40, 2225-2234.

Dale, S.B., Saban, D.R., 2015. Linking immune responses with fibrosis in allergic eye disease. Curr Opin Allergy Clin Immunol 15, 467-475.

Davalos, V., Moutinho, C., Villanueva, A., Boque, R., Silva, P., Carneiro, F., Esteller, M., 2012. Dynamic epigenetic regulation of the microRNA-200 family mediates epithelial and mesenchymal transitions in human tumorigenesis. Oncogene 31, 2062-2074.

Derrick, T., Ramadhani, A.M., Mtengai, K., Massae, P., Burton, M.J., Holland, M.J., 2017. miRNAs that associate with conjunctival inflammation and ocular Chlamydia trachomatis infection do not predict progressive disease. Pathog Dis 75.

Engelsvold, D.H., Utheim, T.P., Olstad, O.K., Gonzalez, P., Eidet, J.R., Lyberg, T., Troseid, A.M.S., Dartt, D.A., Raeder, S., 2013. miRNA and mRNA expression profiling identifies members of the miR-200 family as potential regulators of epithelial-mesenchymal transition in pterygium. Experimental Eye Research 115, 189-198.

Exposito-Villen, A., A, E.A., Franco, D., 2018. Functional Role of Non-Coding RNAs during EpithelialTo-Mesenchymal Transition. Noncoding RNA 4.

Friedlander, M., 2007. Fibrosis and diseases of the eye. J Clin Invest 117, 576-586.

Fu, S.H., Sun, L., Zhang, X.Y., Shi, H.M., Xu, K., Xiao, Y.Q., Ye, W., 2017. 5-Aza-2 '-deoxycytidine induces human Tenon's capsule fibroblasts differentiation and fibrosis by up-regulating TGF-beta type I receptor. Experimental Eye Research 165, 47-58.

Gao, W., Zhang, C.M., Li, W.Q., Li, H.Z., Sang, J.W., Zhao, Q.L., Bo, Y.F., Luo, H.J., Zheng, X.W., Lu, Y., Shi, Y., Yang, D.L., Zhang, R.P., Li, Z.Y., Cui, J.J., Zhang, Y.L., Niu, M., Li, J., Wu, Z.Q., Guo, H.N., Xiang, C.X., Wang, J., Hou, J., Zhang, L., Thorne, R.F., Cui, Y.P., Wu, Y.Y., Wen, S.X., Wang, B.Q., 2019. Promoter Methylation-Regulated miR-145-5p Inhibits Laryngeal Squamous Cell Carcinoma Progression by Targeting FSCN1. Mol Ther 27, 365-379.

Gipson, I.K., Spurr-Michaud, S., Argueso, P., Tisdale, A., Ng, T.F., Russo, C.L., 2003. Mucin gene expression in immortalized human corneal-limbal and conjunctival epithelial cell lines. Invest Ophthalmol Vis Sci 44, 2496-2506.

Gregory, P.A., Bert, A.G., Paterson, E.L., Barry, S.C., Tsykin, A., Farshid, G., Vadas, M.A., KhewGoodall, Y., Goodall, G.J., 2008a. The mir-200 family and mir-205 regulate epithelial to mesenchymal transition by targeting ZEB1 and SIP1. Nat Cell Biol 10, 593-601.

Gregory, P.A., Bracken, C.P., Bert, A.G., Goodall, G.J., 2008b. MicroRNAs as regulators of epithelialmesenchymal transition. Cell Cycle 7, 3112-3117.

Gregory, P.A., Bracken, C.P., Smith, E., Bert, A.G., Wright, J.A., Roslan, S., Morris, M., Wyatt, L., Farshid, G., Lim, Y.Y., Lindeman, G.J., Shannon, M.F., Drew, P.A., Khew-Goodall, Y., Goodall, G.J., 2011. An autocrine TGF-beta/ZEB/miR-200 signaling network regulates establishment and maintenance of epithelial-mesenchymal transition. Mol Biol Cell 22, 1686-1698.

Han, X., Yan, S., Weijie, Z., Feng, W., Liuxing, W., Mengquan, L., Qingxia, F., 2014. Critical role of miR$10 \mathrm{~b}$ in transforming growth factor-beta1-induced epithelial-mesenchymal transition in breast cancer. Cancer Gene Ther 21, 60-67.

Hashimoto, Y., Akiyama, Y., Yuasa, Y., 2013. Multiple-to-Multiple Relationships between MicroRNAs and Target Genes in Gastric Cancer. Plos One 8.

He, S.K., Li, X.H., Chan, N., Hinton, D.R., 2013. Review: Epigenetic mechanisms in ocular disease. Mol Vis 19, 665-674.

Ishikawa, K., He, S., Terasaki, H., Nazari, H., Zhang, H., Spee, C., Kannan, R., Hinton, D.R., 2015.

Resveratrol inhibits epithelial-mesenchymal transition of retinal pigment epithelium and development of proliferative vitreoretinopathy. Sci Rep 5, 16386.

Jiang, J., Yi, B.O., Ding, S., Sun, J., Cao, W., Liu, M., 2016. Demethylation drug 5-Aza-2'-deoxycytidineinduced upregulation of miR-200c inhibits the migration, invasion and epithelial-mesenchymal transition of clear cell renal cell carcinoma in vitro. Oncol Lett 11, 3167-3172. 
Kato, N., Shimmura, S., Kawakita, T., Miyashita, H., Ogawa, Y., Yoshida, S., Higa, K., Okano, H., Tsubota, K., 2007. Beta-catenin activation and epithelial-mesenchymal transition in the pathogenesis of pterygium. Invest Ophthalmol Vis Sci 48, 1511-1517.

Kechagia, J.Z., Ezra, D.G., Burton, M.J., Bailly, M., 2016. Fibroblasts profiling in scarring trachoma identifies IL- 6 as a functional component of a fibroblast-macrophage pro-fibrotic and proinflammatory feedback loop. Sci Rep 6, 28261.

Khaw, P., Grehn, F., Hollo, G., Overton, B., Wilson, R., Vogel, R., Smith, Z., 2007. A phase III study of subconjunctival human anti-transforming growth factor beta(2) monoclonal antibody (CAT-152) to prevent scarring after first-time trabeculectomy. Ophthalmology 114, 1822-1830.

Kim, K.W., Park, S.H., Kim, J.C., 2016. Fibroblast biology in pterygia. Exp Eye Res 142, 32-39.

Kolesnikoff, N., Attema, J.L., Roslan, S., Bert, A.G., Schwarz, Q.P., Gregory, P.A., Goodall, G.J., 2014. Specificity Protein 1 ( Sp1) Maintains Basal Epithelial Expression of the miR-200 Family. Journal of Biological Chemistry 289, 11194-11205.

Lamouille, S., Xu, J., Derynck, R., 2014. Molecular mechanisms of epithelial-mesenchymal transition. Nat Rev Mol Cell Bio 15, 178-196.

Lee, E., Wang, J.C., Yumoto, K., Jung, Y.H., Cackowski, F.C., Decker, A.M., Li, Y., Franceschi, R.T., Pienta, K.J., Taichman, R.S., 2016. DNMT1 Regulates Epithelial-Mesenchymal Transition and Cancer Stem Cells, Which Promotes Prostate Cancer Metastasis. Neoplasia 18, 553-566.

Liu, S., Sun, J., Lan, Q., 2013. TGF-beta-induced miR10a/b expression promotes human glioma cell migration by targeting PTEN. Mol Med Rep 8, 1741-1746.

Long, X.C., Miano, J.M., 2011. Transforming Growth Factor-beta 1 (TGF-beta 1) Utilizes Distinct Pathways for the Transcriptional Activation of MicroRNA 143/145 in Human Coronary Artery Smooth Muscle Cells. Journal of Biological Chemistry 286, 30119-30129.

Lowry, O.H., Rosebrough, N.J., Farr, A.L., Randall, R.J., 1951. Protein measurement with the Folin phenol reagent. J Biol Chem 193, 265-275.

Luo, G.Y., Jing, X., Yang, S., Peng, D.W., Dong, J., Li, L., Reinach, P.S., Yan, D.S., 2019. DNA

Methylation Regulates Corneal Epithelial Wound Healing by Targeting miR-200a and CDKN2B. Invest Ophth Vis Sci 60, 650-660.

McDonald, O.G., Wu, H., Timp, W., Doi, A., Feinberg, A.P., 2011. Genome-scale epigenetic reprogramming during epithelial-to-mesenchymal transition. Nat Struct Mol Biol 18, 867-U821. Micallef, L., Vedrenne, N., Billet, F., Coulomb, B., Darby, I.A., Desmouliere, A., 2012. The myofibroblast, multiple origins for major roles in normal and pathological tissue repair. Fibrogenesis Tissue Repair 5, S5.

Miyazono, K., 2009. Transforming growth factor-beta signaling in epithelial-mesenchymal transition and progression of cancer. Proc Jpn Acad Ser B Phys Biol Sci 85, 314-323.

Nam, J.S., Ino, Y., Kanai, Y., Sakamoto, M., Hirohashi, S., 2004. 5-aza-2'-deoxycytidine restores the Ecadherin system in E-cadherin-silenced cancer cells and reduces cancer metastasis. Clin Exp Metastasis 21, 49-56.

Ogawa, Y., Shimmura, S., Kawakita, T., Yoshida, S., Kawakami, Y., Tsubota, K., 2009. Epithelial mesenchymal transition in human ocular chronic graft-versus-host disease. Am J Pathol 175, 23722381.

Park, S.M., Gaur, A.B., Lengyel, E., Peter, M.E., 2008. The miR-200 family determines the epithelial phenotype of cancer cells by targeting the E-cadherin repressors ZEB1 and ZEB2. Gene Dev 22, 894907.

Pieraccioli, M., Imbastari, F., Antonov, A., Melino, G., Raschella, G., 2013. Activation of miR200 by cMyb depends on ZEB1 expression and miR200 promoter methylation. Cell Cycle 12, 2309-2320.

Pistore, C., Giannoni, E., Colangelo, T., Rizzo, F., Magnani, E., Muccillo, L., Giurato, G., Mancini, M., Rizzo, S., Riccardi, M., Sahnane, N., Del Vescovo, V., Kishore, K., Mandruzzato, M., Macchi, F., Pelizzola, M., Denti, M.A., Furlan, D., Weisz, A., Colantuoni, V., Chiarugi, P., Bonapace, I.M., 2017. DNA methylation variations are required for epithelial-to-mesenchymal transition induced by cancer-associated fibroblasts in prostate cancer cells. Oncogene 36, 5551-5566. 
Raghunath, A., Perumal, E., 2015. Micro-RNAs and Their Roles in Eye Disorders. Ophthalmic Res 53, 169-186.

Rajic, J., Inic-Kanada, A., Stein, E., Dinic, S., Schuerer, N., Uskokovic, A., Ghasemian, E., Mihailovic, M., Vidakovic, M., Grdovic, N., Barisani-Asenbauer, T., 2017. Chlamydia trachomatis Infection Is Associated with E-Cadherin Promoter Methylation, Downregulation of E-Cadherin Expression, and Increased Expression of Fibronectin and alpha-SMA-Implications for Epithelial-Mesenchymal Transition. Front Cell Infect Microbiol 7, 253.

Rheinwald, J.G., Hahn, W.C., Ramsey, M.R., Wu, J.Y., Guo, Z., Tsao, H., De Luca, M., Catricala, C., O'Toole, K.M., 2002. A two-stage, p16(INK4A)- and p53-dependent keratinocyte senescence mechanism that limits replicative potential independent of telomere status. Mol Cell Biol 22, 51575172.

Riau, A.K., Wong, T.T., Lan, W., Finger, S.N., Chaurasia, S.S., Hou, A.H., Chen, S., Yu, S.J., Tong, L., 2011. Aberrant DNA methylation of matrix remodeling and cell adhesion related genes in pterygium. Plos One 6, e14687.

Rout-Pitt, N., Farrow, N., Parsons, D., Donnelley, M., 2018. Epithelial mesenchymal transition (EMT): a universal process in lung diseases with implications for cystic fibrosis pathophysiology. Respir Res $19,136$.

Saika, S., Liu, C.Y., Azhar, M., Sanford, L.P., Doetschman, T., Gendron, R.L., Kao, C.W., Kao, W.W., 2001. TGFbeta2 in corneal morphogenesis during mouse embryonic development. Dev Biol 240, 419432.

Saika, S., Yamanaka, O., Okada, Y., Tanaka, S., Miyamoto, T., Sumioka, T., Kitano, A., Shirai, K., Ikeda, K., 2009. TGF beta in fibroproliferative diseases in the eye. Front Biosci (Schol Ed) 1, 376-390.

Saitoh, M., 2018. Involvement of partial EMT in cancer progression. J Biochem 164, 257-264.

Saw, V.P., Schmidt, E., Offiah, I., Galatowicz, G., Zillikens, D., Dart, J.K., Calder, V.L., Daniels, J.T., 2011. Profibrotic phenotype of conjunctival fibroblasts from mucous membrane pemphigoid. Am J Pathol 178, 187-197.

Schlunck, G., Meyer-ter-Vehn, T., Klink, T., Grehn, F., 2016. Conjunctival fibrosis following filtering glaucoma surgery. Exp Eye Res 142, 76-82.

Serrano-Gomez, S.J., Maziveyi, M., Alahari, S.K., 2016. Regulation of epithelial-mesenchymal transition through epigenetic and post-translational modifications. Mol Cancer 15.

Siriwardena, D., Khaw, P.T., King, A.J., Donaldson, M.L., Overton, B.M., Migdal, C., Cordeiro, M.F., 2002. Human antitransforming growth factor beta(2) monoclonal antibody--a new modulator of wound healing in trabeculectomy: a randomized placebo controlled clinical study. Ophthalmology 109, 427-431.

Stone, R.C., Pastar, I., Ojeh, N., Chen, V., Liu, S., Garzon, K.I., Tomic-Canic, M., 2016. Epithelialmesenchymal transition in tissue repair and fibrosis. Cell Tissue Res 365, 495-506.

Stresemann, C., Lyko, F., 2008. Modes of action of the DNA methyltransferase inhibitors azacytidine and decitabine. Int J Cancer 123, 8-13.

Tam, W.L., Weinberg, R.A., 2013. The epigenetics of epithelial-mesenchymal plasticity in cancer. Nat Med 19, 1438-1449.

Teng, Y.F., Yam, G.H.F., Li, N., Wu, S., Ghosh, A., Wang, N.L., Pang, C.P., Jhanji, V., 2018. MicroRNA regulation of MDM2-p53 loop in pterygium. Experimental Eye Research 169, 149-156.

Tennakoon, A.H., Izawa, T., Kuwamura, M., Yamate, J., 2015. Pathogenesis of Type 2 Epithelial to Mesenchymal Transition (EMT) in Renal and Hepatic Fibrosis. J Clin Med 5.

Thiery, J.P., Acloque, H., Huang, R.Y., Nieto, M.A., 2009. Epithelial-mesenchymal transitions in development and disease. Cell 139, 871-890.

Tsai, Y.Y., Cheng, Y.W., Lee, H., Tsai, F.J., Tseng, S.H., Chang, K.C., 2005. P53 gene mutation spectrum and the relationship between gene mutation and protein levels in pterygium. Mol Vis 11, 50-55.

Wang, Y., Guan, H., 2017. The Role of DNA Methylation in Lens Development and Cataract Formation. Cell Mol Neurobiol 37, 979-984. 
Wu, C.W., Peng, M.L., Yeh, K.T., Tsai, Y.Y., Chiang, C.C., Cheng, Y.W., 2016. Inactivation of p53 in pterygium influence miR-200a expression resulting in ZEB1/ZEB2 up-regulation and EMT processing (vol 146, pg 206, 2016). Experimental Eye Research 151, 256-256.

Xia, W.J., Chen, Q., Wang, J., Mao, Q.X., Dong, G.C., Shi, R., Zheng, Y.Y., Xu, L., Jiang, F., 2015. DNA methylation mediated silencing of microRNA-145 is a potential prognostic marker in patients with lung adenocarcinoma. Sci Rep-Uk 5.

Yan, Y., Wang, Q., Yan, X.L., Zhang, Y., Li, W., Tang, F., Li, X., Yang, P., 2015. miR-10a controls glioma migration and invasion through regulating epithelial-mesenchymal transition via EphA8. FEBS Lett 589, 756-765.

Yoshiura, K., Kanai, Y., Ochiai, A., Shimoyama, Y., Sugimura, T., Hirohashi, S., 1995. Silencing of the Ecadherin invasion-suppressor gene by $\mathrm{CpG}$ methylation in human carcinomas. Proc Natl Acad Sci U S A 92, 7416-7419.

Young, C.H., Chiu, Y.T., Shih, T.S., Lin, W.R., Chiang, C.C., Chou, Y.E., Cheng, Y.W., Tsai, Y.Y., 2010. Ecadherin promoter hypermethylation may contribute to protein inactivation in pterygia. Mol Vis 16 , 1047-1053.

Zada, M., Pattamatta, U., White, A., 2018. Modulation of Fibroblasts in Conjunctival Wound Healing. Ophthalmology 125, 179-192.

Zaravinos, A., 2015. The Regulatory Role of MicroRNAs in EMT and Cancer. J Oncol 2015, 865816. Zeisberg, M., Neilson, E.G., 2009. Biomarkers for epithelial-mesenchymal transitions. J Clin Invest $119,1429-1437$.

Zhang, H.P., Wang, Y.H., Cao, C.J., Yang, X.M., Ma, S.C., Han, X.B., Yang, X.L., Yang, A.N., Tian, J., Xu, H., Zhang, M.H., Jiang, Y.D., 2016. A regulatory circuit involving miR-143 and DNMT3a mediates vascular smooth muscle cell proliferation induced by homocysteine. Molecular Medicine Reports 13, 483-490.

Zhang, L., Sun, J., Wang, B., Ren, J.C., Su, W., Zhang, T., 2015. MicroRNA-10b Triggers the EpithelialMesenchymal Transition (EMT) of Laryngeal Carcinoma Hep-2 Cells by Directly Targeting the Ecadherin. Appl Biochem Biotechnol 176, 33-44.

Zhou, P., Lu, Y., Sun, X.H., 2011. Zebularine suppresses TGF-beta-induced lens epithelial cellmyofibroblast transdifferentiation by inhibiting MeCP2. Mol Vis 17, 2717-2723.

Zhou, P., Lu, Y., Sun, X.H., 2012. Effects of a novel DNA methyltransferase inhibitor Zebularine on human lens epithelial cells. Mol Vis 18, 22-28. 